\title{
Infinitely many solutions for nonlinear fractional boundary value problems via variational methods
}

\section{Guoging Chai*}

\section{"Correspondence:}

mathchgq@163.com

College of Mathematics and

Statistics, Hubei Normal University,

Huangshi, Hubei 435002, P.R. China

\begin{abstract}
In this paper, the author considers the following nonlinear fractional boundary value problem:

$$
\left\{\begin{array}{l}
\frac{d}{d t}\left(\frac{1}{2}{ }_{0} D_{t}^{-\beta}\left(u^{\prime}(t)\right)+\frac{1}{2} t D_{T}^{-\beta}\left(u^{\prime}(t)\right)\right)+\nabla F(t, u(t))=0, \quad \text { a.e. } t \in[0, T] \\
u(0)=u(T)=0,
\end{array}\right.
$$

where ${ }_{0} D_{t}^{-\beta}$ and ${ }_{t} D_{T}^{-\beta}$ are the left and right Riemann-Liouville fractional integrals of order $0 \leq \beta<1$, respectively, $\nabla F(t, x)$ is the gradient of $F$ at $x$. By applying the variant fountain theorems, the author obtains the existence of infinitely many small or high energy solutions to the above boundary value problem.
\end{abstract}

Keywords: fractional differential equations; variant fountain theorems; critical point theory; variational method

\section{Introduction}

Fractional calculus has applications in many areas, including fluid flow, electrical networks, probability and statistics, chemical physics and signal processing and so on; see [1-6] and the references therein. In recent years, there have been many papers dealing with the existence of solutions of nonlinear initial (or boundary) value problems of fractional equations by applying nonlinear analysis such as fixed point theorems, lower and upper solution method, monotone iterative method, coincidence degree theory. However, up to now, there are few results on the solutions to fractional boundary value problems that are established by the variational methods; see for example, [7-20]. It is often very difficult to establish a suitable space and variational functional for fractional boundary value problem, especially for the fractional equations including both left and right fractional derivatives.

Jiao and Zhou [7] were first to show that the critical point theory is an effective approach to track the existence of solutions to the following fractional boundary value problem (BVP for short):

$$
\left\{\begin{array}{l}
\frac{d}{d t}\left(\frac{1}{2}{ }_{0} D_{t}^{-\beta}\left(u^{\prime}(t)\right)+\frac{1}{2}{ }_{t} D_{T}^{-\beta}\left(u^{\prime}(t)\right)\right)+\nabla F(t, u(t))=0, \quad \text { a.e. } t \in[0, T] \\
u(0)=u(T)=0,
\end{array}\right.
$$

(c) 2016 Chai. This article is distributed under the terms of the Creative Commons Attribution 4.0 International License (http://creativecommons.org/licenses/by/4.0/), which permits unrestricted use, distribution, and reproduction in any medium, provided you give appropriate credit to the original author(s) and the source, provide a link to the Creative Commons license, and indicate if changes were made. 
where ${ }_{0} D_{t}^{-\beta}$ and ${ }_{t} D_{T}^{-\beta}$ are the left and right Riemann-Liouville fractional integrals of order $0 \leq \beta<1$, respectively, $\nabla F(t, x)$ is the gradient of $F$ at $x$.

As in [7], for any $u \in A C\left([0, T], \mathbb{R}^{N}\right)$, BVP (1.1) is equivalent to the following problem:

$$
\left\{\begin{array}{l}
\frac{d}{d t}\left(\frac{1}{2}{ }_{0} D_{t}^{\alpha-1}\left({ }_{0}^{c} D_{t}^{\alpha} u(t)\right)-\frac{1}{2} t D_{T}^{\alpha-1}\left({ }_{t}^{c} D_{T}^{\alpha} u(t)\right)\right)+\nabla F(t, u(t))=0, \quad \text { a.e. } t \in[0, T], \\
u(0)=u(T)=0
\end{array}\right.
$$

where $\alpha=1-\beta / 2 \in(1 / 2,1]$.

Physical models containing fractional differential operators have recently renewed attention from scientists which is mainly due to applications as models for physical phenomena exhibiting anomalous diffusion. A strong motivation for investigating the fractional BVP (1.1) comes from the fractional advection-dispersion equation (ADE). A fractional $\mathrm{ADE}$ is a generalization of the classical $\mathrm{ADE}$ in which the second-order derivative is replaced with a fractional-order derivative. In contrast to the classical ADE, the fractional ADE has solutions that resemble the highly skewed and heavy-tailed breakthrough curves observed in field and laboratory studies [21,22], in particular in contaminant transport of groundwater flow [23]. In [23], Benson et al. stated that solutes moving through a highly heterogeneous aquifer violate the basic assumptions of local second-order theories because of large deviations from the stochastic process of Brownian motion.

In [7], Jiao and Zhou obtained the existence of solutions for BVP (1.1) by the mountain pass theorem under the Ambrosetti-Rabinowitz condition. Following Jiao's work [7-16] studied the BVP (1.1) or its variant form. In [8, 9], Chen and Tang studied the existence and multiplicity of solutions by use of the mountain pass theorem or the fountain theorem. In [10-12], by use of some three critical points theorem or the mountain pass theorem, the authors, respectively, studied the existence of multiple solutions to the following problem:

$$
\left\{\begin{array}{l}
\frac{d}{d t}\left(\frac{1}{2}{ }_{0} D_{t}^{-\beta}\left(u^{\prime}(t)\right)+\frac{1}{2} t D_{T}^{-\beta}\left(u^{\prime}(t)\right)\right)+\lambda \nabla F(t, u(t))=0, \quad \text { a.e. } t \in[0, T], \\
u(0)=u(T)=0,
\end{array}\right.
$$

where $\lambda$ is a parameter. In [13], by using the critical point theorem established by Bonanno, Bai investigated the following problem:

$$
\left\{\begin{array}{l}
\frac{d}{d t}\left(\frac{1}{2}{ }_{0} D_{t}^{-\beta}\left(u^{\prime}(t)\right)+\frac{1}{2} t_{T}^{-\beta}\left(u^{\prime}(t)\right)\right)+\lambda a(t) f(u(t))=0, \quad \text { a.e. } t \in[0, T], \\
u(0)=u(T)=0
\end{array}\right.
$$

where $\lambda$ is a parameter and $a: \mathbb{R} \rightarrow \mathbb{R}$ is a nonnegative continuous function. In [14], by applying critical point theorems, Li, Sun and Zhang studied the existence of solutions to the following problem:

$$
\left\{\begin{array}{l}
-\frac{d}{d t}\left(\frac{1}{2} 0 D_{t}^{-\beta}\left(u^{\prime}(t)\right)+\frac{1}{2} t D_{T}^{-\beta}\left(u^{\prime}(t)\right)\right)=\lambda u(t)+\nabla F(t, u(t)), \quad \text { a.e. } t \in[0, T], \\
u(0)=u(T)=0
\end{array}\right.
$$

where $\lambda$ lies various interval. In [15], by the variational method combined with an iterative technique, Sun and Zhang investigated the existence of solutions to the following problems:

$$
\left\{\begin{array}{l}
\frac{d}{d t}\left(p_{0} D_{t}^{-\beta}\left(u^{\prime}(t)\right)+q_{t} D_{1}^{-\beta}\left(u^{\prime}(t)\right)\right)+f(t, u(t))=0, \quad t \in[0,1] \\
u(0)=u(1)=0
\end{array}\right.
$$


Very recently, in [16], by using a three critical points theorem, Ferrara and Hadjian studied the existence of three solutions for the following problem:

$$
\left\{\begin{array}{l}
\frac{d}{d t}\left(\frac{1}{2}{ }_{0} D_{t}^{-\beta}\left(u^{\prime}(t)\right)+\frac{1}{2}{ }_{t} D_{T}^{-\beta}\left(u^{\prime}(t)\right)\right)+\lambda f(t, u(t))+\mu g(t, u(t))=0, \quad \text { a.e. } t \in[0, T] \\
u(0)=u(T)=0
\end{array}\right.
$$

where $\lambda, \mu$ are two parameters and $f, g:[0, T] \times \mathbb{R} \rightarrow \mathbb{R}$ are two nonnegative continuous functions.

Some fractional equations including both left and right fractional derivatives and having some relations with BVP (1.1) were also investigated; see, for example, [17-20]. By the critical point theory, Jiao and Zhou in [17] established the existence of solutions for the following fractional boundary value problem:

$$
\left\{\begin{array}{l}
{ }_{t} D_{T}^{\alpha}\left({ }_{0} D_{t}^{\alpha} u(t)\right)=\nabla F(t, u(t)), \quad \text { a.e. } t \in[0,1] \\
u(0)=u(T)=0
\end{array}\right.
$$

On the other hand, the variant fountain theorems are effective tools for studying the existence of infinitely many high or small energy solutions [24, 25].

Different from the work mentioned above, in this paper, the author attempts to apply the variant fountain theorems to study the existence of infinitely many small or high energy solutions to BVP (1.1). As pointed out in [24, 25], the variant fountain theorems do not need the (P.S.) or (P.S.*) conditions, which is an important condition usually assumed in the literature. By taking advantage of the variant fountain theorems, to consider the infinitely many solutions to BVP (1.1) in this paper, the Ambrosetti-Rabinowitz condition is not needed. This is one of the new features of the paper compared with some papers above such as [9]. In [9], because of applying fountain theorems, the (P.S.) condition, or say, the Ambrosetti-Rabinowitz condition (which ensures that the P.S. condition holds) is necessary. In addition, the assumed conditions in this paper are easy to verify.

The paper is arranged as follows. In Section 2, the author presents some necessary preliminary facts that will be needed in the paper. In Section 3, the author establishes the existence of infinitely many small or high energy solutions for BVP (1.1) and gives two examples to show the effectiveness of the results obtained.

\section{Preliminaries}

To apply the variant fountain theorems to the existence of infinitely many solutions for BVP (1.1), we shall state some basic notations and results, which will be used in the proofs of our main results.

Throughout this paper, we denote $\alpha=1-\frac{\beta}{2}$, and we assume that the following condition is satisfied.

(H) $F(t, x)$ is measurable in $t$ for every $x \in \mathbb{R}^{N}$ and continuously differentiable in $x$ for a.e. $t \in[0, T]$, and there exists $a \in C\left(\mathbb{R}^{+}, \mathbb{R}^{+}\right), b \in L\left([0, T], \mathbb{R}^{+}\right)$such that

$$
|F(t, x)| \leq a(|x|) b(t), \quad|\nabla F(t, x)| \leq a(|x|) b(t)
$$

for all $x \in \mathbb{R}^{N}$ and a.e. $t \in[0, T]$. 
Definition 2.1 ([6]) Let $f$ be a function defined on $[a, b]$. The left and right RiemannLiouville fractional integrals of order $\gamma$ for function $f$ denoted by ${ }_{a} D_{t}^{-\gamma} f(t)$ and ${ }_{t} D_{b}^{-\gamma} f(t)$, respectively, are defined by

$$
{ }_{a} D_{t}^{-\gamma} f(t)=\frac{1}{\Gamma(\gamma)} \int_{a}^{t}(t-s)^{\gamma-1} f(s) d s, \quad t \in[a, b], \gamma>0
$$

and

$$
{ }_{t} D_{b}^{-\gamma} f(t)=\frac{1}{\Gamma(\gamma)} \int_{t}^{b}(s-t)^{\gamma-1} f(s) d s, \quad t \in[a, b], \gamma>0,
$$

provided in both cases that the right-hand side is pointwise defined on $[a, b]$, where $\Gamma$ is the gamma function.

Definition 2.2 ([6]) Let $f$ be a function defined on $[a, b]$. The left and right RiemannLiouville fractional derivatives of order $\gamma$ for function $f$ denoted by ${ }_{a} D_{t}^{\gamma} f(t)$ and ${ }_{t} D_{b}^{\gamma} f(t)$, respectively, exist almost everywhere on $[a, b] .{ }_{a} D_{t}^{\gamma} f(t)$ and ${ }_{t} D_{b}^{\gamma} f(t)$ are represented by

$$
{ }_{a} D_{t}^{\gamma} f(t)=\frac{1}{\Gamma(n-\gamma)} \frac{d^{n}}{d t^{n}} \int_{a}^{t}(t-s)^{n-\gamma-1} f(s) d s, \quad t \in[a, b]
$$

and

$$
{ }_{t} D_{b}^{\gamma} f(t)=\frac{(-1)^{n}}{\Gamma(n-\gamma)} \frac{d^{n}}{d t^{n}} \int_{t}^{b}(s-t)^{n-\gamma-1} f(s) d s, \quad t \in[a, b]
$$

where $n-1 \leq \gamma<n$ and $n \in \mathbb{N}$. In particular, if $0 \leq \gamma<1$, then

$$
{ }_{a} D_{t}^{\gamma} f(t)=\frac{1}{\Gamma(1-\gamma)} \frac{d}{d t} \int_{a}^{t}(t-s)^{-\gamma} f(s) d s, \quad t \in[a, b]
$$

and

$$
{ }_{t} D_{b}^{\gamma} f(t)=-\frac{1}{\Gamma(1-\gamma)} \frac{d}{d t} \int_{t}^{b}(s-t)^{-\gamma} f(s) d s, \quad t \in[a, b] .
$$

Definition 2.3 ([6]) If $\gamma \in(n-1, n)$ and $f \in A C^{n}\left([a, b], \mathbb{R}^{\mathrm{N}}\right)$, then the left and right Caputo fractional derivatives of order $\gamma$ for function $f$ denoted by ${ }_{a}^{c} D_{t}^{\gamma} f(t)$ and ${ }_{t}^{c} D_{b}^{\gamma} f(t)$, respectively, exist almost everywhere on $[a, b] .{ }_{a}^{c} D_{t}^{\gamma} f(t)$ and ${ }_{t}^{c} D_{b}^{\gamma} f(t)$ are represented by

$$
{ }_{a}^{c} D_{t}^{\gamma} f(t)={ }_{a} D_{t}^{\gamma-n} f^{(n)}(t)=\frac{1}{\Gamma(n-\gamma)} \int_{a}^{t}(t-s)^{n-\gamma-1} f^{(n)}(s) d s
$$

and

$$
{ }_{t}^{c} D_{b}^{\gamma} f(t)=(-1)^{n}{ }_{t} D_{b}^{\gamma-n} f^{(n)}(t)=\frac{(-1)^{n}}{\Gamma(n-\gamma)} \int_{t}^{b}(s-t)^{n-\gamma-1} f^{(n)}(s) d s,
$$

respectively, where $t \in[a, b]$. In particular, if $0<\gamma<1$, then

$$
{ }_{a}^{c} D_{t}^{\gamma} f(t)={ }_{a} D_{t}^{\gamma-1} f^{\prime}(t)=\frac{1}{\Gamma(1-\gamma)} \int_{a}^{t}(t-s)^{-\gamma} f^{\prime}(s) d s, \quad t \in[a, b]
$$


and

$$
{ }_{t}^{c} D_{b}^{\gamma} f(t)=-{ }_{t} D_{b}^{\gamma-1} f^{\prime}(t)=-\frac{1}{\Gamma(1-\gamma)} \int_{t}^{b}(s-t)^{-\gamma} f^{\prime}(s) d s, \quad t \in[a, b] .
$$

Let us recall that for any $u \in L^{p}[0, T], 1 \leq p<\infty,\|u\|_{p}=\left(\int_{0}^{T}|u(t)|^{p} d t\right)^{1 / p}$, and $u \in$ $C[0, T],\|u\|_{\infty}=\max _{t \in[0, T]}|u(t)|$.

Definition 2.4 Let $0<\alpha \leq 1$ and $1<p<\infty$. The fractional derivative space $E_{0}^{\alpha, p}$ is defined by the closure of $C_{0}^{\infty}\left([0, T], \mathbb{R}^{N}\right)$ with respect to the weighted norm

$$
\|u\|_{\alpha, p}=\left(\int_{0}^{T}|u(t)|^{p} d t+\int_{0}^{T}\left|{ }_{0}^{c} D_{t}^{\alpha} u(t)\right|^{p} d t\right)^{1 / p}, \quad \forall u \in E_{0}^{\alpha, p} .
$$

As in [7], we note the following.

\section{Remark 2.1}

(1) The fractional derivative space $E_{0}^{\alpha, p}$ is the space of functions $u \in L^{p}\left([0, T], \mathbb{R}^{N}\right)$ having an $\alpha$ th order Caputo fractional derivative ${ }_{0}^{c} D_{t}^{\alpha} u \in L^{p}\left([0, T], \mathbb{R}^{N}\right)$ and $u(0)=u(T)=0$.

(2) For any $u \in E_{0}^{\alpha, p}$, noting the fact that $u(0)=0$, we have ${ }_{0}^{c} D_{t}^{\alpha} u(t)={ }_{0} D_{t}^{\alpha} u(t), t \in[0, T]$.

Lemma 2.1 ([7]) Let $0<\alpha \leq 1$ and $1<p<\infty$. The fractional derivative space $E_{0}^{\alpha, p}$ is a reflexive and separable Banach space.

Lemma 2.2 ([7]) Let $0<\alpha \leq 1$ and $1<p<\infty$. For any $u \in E_{0}^{\alpha, p}$, we have

$$
\|u\|_{p} \leq \frac{T^{\alpha}}{\Gamma(\alpha+1)}\left\|{ }_{0}^{c} D_{t}^{\alpha} u\right\|_{p}
$$

Moreover, if $\alpha>1 / p$ and $1 / p+1 / q=1$, then

$$
\|u\|_{\infty} \leq \frac{T^{\alpha-1 / p}}{\Gamma(\alpha)((\alpha-1) q+1)^{1 / q}}\left\|{ }_{0}^{c} D_{t}^{\alpha} u\right\|_{p} .
$$

According to Lemma 2.2, we consider $E_{0}^{\alpha, p}$ with respect to the norm

$$
\|u\|_{\alpha, p}=\left\|{ }_{0}^{c} D_{t}^{\alpha} u\right\|_{p}=\left(\int_{0}^{T}\left|{ }_{0}^{c} D_{t}^{\alpha} u(t)\right|^{p} d t\right)^{1 / p}
$$

in this paper.

Lemma 2.3 ([7]) Let $0<\alpha \leq 1$ and $1<p<\infty$. If $\alpha>\frac{1}{p}$ and the sequence $\left\{u_{k}\right\}$ converges weakly to $u$ in $E_{0}^{\alpha, p}$, i.e. $u_{k} \rightarrow u$, then $u_{k} \rightarrow u$ in $C([0, T])$, i.e. $\left\|u-u_{k}\right\|_{\infty} \rightarrow 0$ as $k \rightarrow \infty$.

In the following, we always consider the space $E_{0}^{\alpha, p}$ with $p=2$ and denote $E^{\alpha}=E_{0}^{\alpha, 2}$ with the corresponding norm $\|u\|=\|u\|_{\alpha, 2}$. Moreover, we always assume that $\frac{1}{2}<\alpha \leq 1$. 
Remark 2.2 By Lemma 2.1, it is easy to see that the space $E^{\alpha}$ is a separable Hilbert space with the inner product

$$
\langle u, v\rangle=\int_{0}^{T}\left({ }_{0}^{c} D_{t}^{\alpha} u(t),{ }_{0}^{c} D_{t}^{\alpha} v(t)\right) d t, \quad u, v \in E^{\alpha}
$$

Lemma 2.4 ([7]) If $1 / 2<\alpha \leq 1$, then for any $u \in E^{\alpha}$, we have

$$
|\cos (\pi \alpha)|\|u\|^{2} \leq-\int_{0}^{T}\left({ }_{0}^{c} D_{t}^{\alpha} u(t),{ }_{t}^{c} D_{T}^{\alpha} u(t)\right) d t \leq \frac{1}{|\cos (\pi \alpha)|}\|u\|^{2} .
$$

Let us denote $D^{\alpha} u(t)=\frac{1}{2}{ }_{0} D_{t}^{\alpha-1}\left({ }_{0}^{c} D_{t}^{\alpha} u(t)\right)-\frac{1}{2} t D_{T}^{\alpha-1}\left({ }_{t}^{c} D_{T}^{\alpha} u(t)\right)$.

Definition 2.5 A function $u \in A C\left([0, T], \mathbb{R}^{N}\right)$ is called a solution of BVP (1.2) if

(1) $D^{\alpha} u(t)$ is derivable for almost every $t \in[0, T]$, and

(2) $u$ satisfies (1.2).

Remark 2.3 As before, for $\alpha=1-\beta / 2$ with $1 / 2<\alpha \leq 1$, if $u \in A C\left([0, T], \mathbb{R}^{N}\right)$ is a solution of BVP (1.1) iff $u$ is a solution of BVP (1.2).

To study the existence of infinitely many solutions of BVP (1.2), we need to introduce the following variant fountain theorems. Let $X$ be a Banach space with the space norm $\|\cdot\|$, and $X=\overline{\bigoplus_{j \in \mathbb{N}} X_{j}}$ with $\operatorname{dim} X_{j}<\infty$ for any $j \in \mathbb{N}$. Set $W_{k}=\bigoplus_{j=1}^{k} X_{j}, Z_{k}=\overline{\bigoplus_{j=k}^{\infty} X_{j}}$, and $B_{k}=\left\{u \in W_{k}:\|u\| \leq \rho_{k}\right\}, S_{k}=\left\{u \in Z_{k}:\|u\|=r_{k}\right\}$. For $\rho_{k}>r_{k}>0$, consider a family of $C^{1}-$ functionals $\Phi_{\lambda}: X \rightarrow \mathbb{R}$ defined by

$$
\Phi_{\lambda}(u)=A(u)-\lambda B(u), \quad \lambda \in[1,2] .
$$

The following two variant fountain theorems were established in $[24,25]$.

Lemma 2.5 Assume that $\Phi_{\lambda}$ satisfies:

(A $\left.\Phi_{1}\right) \Phi_{\lambda}$ maps bounded sets into bounded sets uniformly for $\lambda \in[1,2]$, and $\Phi_{\lambda}(-u)=\Phi_{\lambda}(u)$ for all $(\lambda, u) \in[1,2] \times X$;

$\left(\mathrm{A}_{2}\right) B(u) \geq 0$ for all $u \in X$, and $B(u) \rightarrow \infty$ as $\|u\| \rightarrow \infty$ on any finite-dimensional subspace of $X$;

$\left(\mathrm{A}_{3}\right)$ there exists $\rho_{k}>r_{k}>0$ such that

$$
\begin{aligned}
& a_{k}(\lambda)=\inf _{u \in Z_{k},\|u\|=\rho_{k}} \Phi_{\lambda}(u) \geq 0, \\
& b_{k}(\lambda)=\max _{u \in W_{k},\|u\|=r_{k}} \Phi_{\lambda}(u)<0, \quad \forall \lambda \in[1,2], \\
& d_{k}(\lambda)=\inf _{u \in Z_{k},\|u\| \leq \rho_{k}} \Phi_{\lambda}(u) \rightarrow 0 \quad \text { as } k \rightarrow \infty \text { uniformly } \lambda \in[1,2] .
\end{aligned}
$$

Then there exist $\lambda_{n} \rightarrow 1, u_{n}\left(\lambda_{n}\right) \in W_{n}$ such that

$$
\left.\Phi_{\lambda_{n}}^{\prime}\left(u\left(\lambda_{n}\right)\right)\right|_{W_{n}}=0 \text { and } \Phi_{\lambda_{n}}\left(u\left(\lambda_{n}\right)\right) \rightarrow c_{k} \quad \text { as } n \rightarrow \infty,
$$


where $c_{k} \in\left[d_{k}(2), b_{k}(1)\right]$. In particular, if $\left\{u\left(\lambda_{n}\right)\right\}$ has a convergent subsequence for every $k$, then $\Phi_{1}$ has infinitely many nontrivial critical points $\left\{u_{k}\right\} \in X \backslash\{0\}$ satisfying $\Phi_{1}\left(u_{k}\right) \rightarrow 0^{-}$ as $n \rightarrow \infty$.

Lemma 2.6 Assume that the functional $\Phi_{\lambda}$ defined above satisfies:

(B) $\Phi_{\lambda}$ maps bounded sets into bounded sets uniformly for $\lambda \in[1,2]$, and $\Phi_{\lambda}(-u)=\Phi_{\lambda}(u)$ for all $(\lambda, u) \in[1,2] \times X$;

$\left(\mathrm{B}_{2}\right) \quad B(u) \geq 0$ for all $u \in X, A(u) \rightarrow \infty$ or $B(u) \rightarrow \infty$ as $\|u\| \rightarrow \infty$; or

(B) $B(u) \leq 0$ for all $u \in X, B(u) \rightarrow-\infty$ as $\|u\| \rightarrow \infty$;

$\left(\mathrm{B}_{4}\right)$ there exists $\rho_{k}>r_{k}>0$ such that

$$
b_{k}(\lambda)=\inf _{u \in Z_{k},\|u\|=r_{k}} \Phi_{\lambda}(u)>a_{k}(\lambda)=\max _{u \in W_{k},\|u\|=\rho_{k}} \Phi_{\lambda}(u), \quad \forall \lambda \in[1,2] .
$$

Then

$$
b_{k}(\lambda) \leq c_{k}(\lambda)=\inf _{\gamma \in \Gamma_{k}} \max _{u \in B_{k}} \Phi_{\lambda}(\gamma(u)), \quad \forall \lambda \in[1,2]
$$

where $\Gamma_{k}=\left\{\gamma \in C\left(B_{k}, X\right): \gamma\right.$ is odd, $\left.\left.\gamma\right|_{\partial B_{k}}=\mathrm{id}\right\}(k \geq 3)$. Moreover, for almost every $\lambda \in$ $[1,2]$, there exists a sequence $\left\{u_{n}^{k}(\lambda)\right\}$ such that

$$
\sup _{n}\left\|u_{n}^{k}(\lambda)\right\|<\infty, \quad \Phi_{\lambda}^{\prime}\left(u_{n}^{k}(\lambda)\right) \rightarrow 0 \quad \text { and } \quad \Phi_{\lambda}\left(u_{n}^{k}(\lambda)\right) \rightarrow c_{k}(\lambda) \quad \text { as } n \rightarrow \infty .
$$

Remark 2.4 Carefully analyzing the proof of Lemma 2.5 and Lemma 2.6 in [24, 25], we can find that the condition $\left(\mathrm{A}_{3}\right)$ in Lemma 2.5 and the condition $\left(\mathrm{B}_{4}\right)$ may be slightly weak compared to the condition $\left(\mathrm{A}_{3}\right)^{\prime}$ and condition $\left(\mathrm{B}_{4}\right)^{\prime}$, respectively, as follows:

$\left(\mathrm{A}_{3}\right)^{\prime}$ There exist a $k_{0}>0$ and $\rho_{k}>r_{k}>0$ such that for $k \geq k_{0}$ the following relations hold:

$$
\begin{aligned}
& a_{k}(\lambda)=\inf _{u \in Z_{k},\|u\|=\rho_{k}} \Phi_{\lambda}(u) \geq 0, \\
& b_{k}(\lambda)=\max _{u \in W_{k},\|u\|=r_{k}} \Phi_{\lambda}(u)<0, \quad \forall \lambda \in[1,2], \\
& d_{k}(\lambda)=\inf _{u \in Z_{k},\|u\| \leq \rho_{k}} \Phi_{\lambda}(u) \rightarrow 0 \quad \text { as } k \rightarrow \infty \text { uniformly } \lambda \in[1,2] .
\end{aligned}
$$

$\left(\mathrm{B}_{4}\right)^{\prime}$ There exist a $k_{0}>0$ and $\rho_{k}>r_{k}>0$ such that for $k \geq k_{0}$, the following relation holds:

$$
b_{k}(\lambda)=\inf _{u \in Z_{k},\|u\|=r_{k}} \Phi_{\lambda}(u)>a_{k}(\lambda)=\max _{u \in W_{k},\|u\|=\rho_{k}} \Phi_{\lambda}(u), \quad \forall \lambda \in[1,2] .
$$

Then the conclusions in Lemma 2.5 and Lemma 2.6 still hold for $k \geq k_{0}$.

\section{Main results}

We define a family of functionals $\Phi_{\lambda}: E^{\alpha} \rightarrow \mathbb{R}$ as follows:

$$
\Phi_{\lambda}(u)=A u-\lambda B u, \quad \lambda \in[1,2],
$$


where

$$
A u=-\frac{1}{2} \int_{0}^{T}\left({ }_{0}^{c} D_{t}^{\alpha} u(t),{ }_{t}^{c} D_{T}^{\alpha} u(t)\right) d t, \quad B u=\int_{0}^{T} F(t, u(t)) d t .
$$

In view of [7], we know that under the condition $(H), \Phi_{\lambda}$ is continuously differential and

$$
\begin{aligned}
\left\langle\Phi_{\lambda}^{\prime}(u), v\right\rangle= & -\frac{1}{2} \int_{0}^{T}\left[\left({ }_{0}^{c} D_{t}^{\alpha} u(t),{ }_{t}^{c} D_{T}^{\alpha} v(t)\right)+\left({ }_{t}^{c} D_{T}^{\alpha} u(t),{ }_{0}^{c} D_{t}^{\alpha} \nu(t)\right)\right] d t \\
& -\lambda \int_{0}^{T}(\nabla F(t, u(t)), v(t)) d t
\end{aligned}
$$

for any $u, v \in E^{\alpha}$.

Definition 3.1 A function $u \in E^{\alpha}$ is called a weak solution of BVP (1.2) if

$$
\begin{aligned}
& \frac{1}{2} \int_{0}^{T}\left[\left({ }_{0}^{c} D_{t}^{\alpha} u(t),{ }_{t}^{c} D_{T}^{\alpha} v(t)\right)+\left({ }_{t}^{c} D_{T}^{\alpha} u(t),{ }_{0}^{c} D_{t}^{\alpha} v(t)\right)\right] d t \\
& +\int_{0}^{T}(\nabla F(t, u(t)), v(t)) d t=0
\end{aligned}
$$

for any $v \in E^{\alpha}$.

By Theorem 4.2 in [7], we have the following lemma.

Lemma 3.1 If $u \in E^{\alpha}$ is a weak solution of $B V P$ (1.2), then $u$ is a solution of $B V P$ (1.2).

Hence, by Remark 2.3, and (3.1)-(3.4), we may concentrate our attention on finding critical points of the functional $\Phi_{1}$ in $E^{\alpha}$ to obtain some solutions of BVP (1.1).

Since $E^{\alpha}$ is a separable Hilbert space in terms of Remark 2.2, we can choose a completely orthonormal basis $\left\{e_{i}\right\}_{1}^{\infty}$ of $E^{\alpha}$ and define $X_{j}=\mathbb{R} e_{j}$. Then $W_{k}, Z_{k}$ can be defined as before.

We give a list of assumptions which will be used in the sequel.

$\left(\mathrm{H}_{1}\right) \quad F(t, x) \geq 0, F(t,-x)=F(t, x)$, for all $x \in \mathbb{R}^{N}$ and a.e. $t \in[0, T]$.

$\left(\mathrm{H}_{2}\right)$ There exist constants $\sigma, d>0$ such that

$$
\lim _{|x| \rightarrow \infty} \frac{F(t, x)}{|x|^{\sigma}}>d \quad \text { for a.e. } t \in[0, T]
$$

$\left(\mathrm{H}_{3}\right)$ There exist constants $0<\tau, \eta<2$, and $\delta>0$, function $l \in L^{p}[0, T](p=2 /(2-\eta))$ with $l(t) \geq 0$ for a.e. $t \in[0, T]$, and function $c$ satisfying that $c(t)>0$ a.e. $t \in[0,1], 0<$ $\int_{0}^{T} c^{-r}(t) d t<\infty$ for some $r>0$ such that

$$
c(t)|x|^{\tau} \leq F(t, x) \leq l(t)|x|^{\eta}
$$

for all $x \in \mathbb{R}^{N}$ with $|x| \leq \delta$ and a.e. $t \in[0, T]$.

$\left(\mathrm{H}_{4}\right)$ There exist constants $\gamma, \mu$ with $\gamma \in(0,2), \mu<1 / 2$ and a function $d \in L^{2 /(2-\gamma)}$ such that

$$
F(t, x)-\mu(\nabla F(t, x), x) \leq d(t)|x|^{\gamma} \quad \text { for all } x \in \mathbb{R}^{N} \text { and a.e. } t \in[0, T] .
$$


We give several lemmas below which will be used in the proof of our main results.

Lemma 3.2 Let $E$ be any finite-dimensional subspace of $E^{\alpha}$. Then there exists a constant $\varepsilon_{0}>0$ such that

$$
\operatorname{meas}\left\{t \in[0, T]|| u(t) \mid \geq \varepsilon_{0}\|u\|\right\} \geq \varepsilon_{0}
$$

for all $u \in E \backslash\{0\}$.

Proof On the contrary, suppose that there exists a sequence $\left\{u_{n}\right\} \subset E \backslash\{0\}$ such that

$$
\operatorname{meas}\left\{t \in[0, T]|| u_{n}(t) \mid \geq \frac{1}{n}\|u\|\right\}<\frac{1}{n}, \quad \forall n \in \mathbb{N} .
$$

Let $v_{n}=\frac{u_{n}}{\left\|u_{n}\right\|}, n \in \mathbb{N}$. Then $\left\|v_{n}\right\|=1$ and

$$
\text { meas }\left\{t \in[0, T]|| v_{n}(t) \mid \geq \frac{1}{n}\right\}<\frac{1}{n}, \quad \forall n \in \mathbb{N} \text {. }
$$

By the boundedness of $\left\{v_{n}\right\}$, passing to a subsequence, if necessary, we may assume that $v_{n} \rightarrow v$ with $\|v\|=1$ in $E$ for some $v \in E$. From the equivalence of any norm in finitedimensional space, it follows that

$$
\int_{0}^{T}\left|v_{n}(t)-v(t)\right|^{2} d t \rightarrow 0 \quad \text { as } n \rightarrow \infty
$$

Since $v \neq 0$, there exists a constant $\delta_{0}>0$ such that

$$
\operatorname{meas}\left\{t \in[0, T]|| v(t) \mid \geq \delta_{0}\right\} \geq \delta_{0}
$$

Let $\Omega_{n}=\left\{t \in[0, T]:\left|v_{n}(t)\right|<\frac{1}{n}\right\}, \Omega_{n}^{c}=\left\{t \in[0, T]:\left|v_{n}(t)\right| \geq \frac{1}{n}\right\}$, and $\Omega_{0}=\{t \in[0, T]|| v(t) \mid \geq$ $\left.\delta_{0}\right\}$. Then for $n$ large enough, from (3.8), (3.6), it follows that

$$
\operatorname{meas}\left(\Omega_{n} \cap \Omega_{0}\right) \geq \operatorname{meas}\left(\Omega_{0}\right)-\operatorname{meas}\left(\Omega_{n}^{c}\right) \geq \frac{2}{3} \delta_{0},
$$

and therefore

$$
\begin{aligned}
\int_{0}^{T}\left|v_{n}(t)-v(t)\right|^{2} d t & \geq \int_{\Omega_{n} \cap \Omega_{0}}\left|v_{n}(t)-v(t)\right|^{2} d t \\
& \geq \int_{\Omega_{n} \cap \Omega_{0}}\left(|v(t)|^{2}-2\left|v_{n}(t)\right||v(t)|\right) d t \\
& \geq \delta_{0}\left(\delta_{0}-\frac{2}{n}\right) \operatorname{meas}\left(\Omega_{n} \cap \Omega_{0}\right) \geq \frac{2}{9} \delta_{0}^{3}>0
\end{aligned}
$$

for $n$ large enough. This is in contradiction with (3.7), and the proof is complete.

By an argument similar to the proof of Lemma 3 in [26], we can obtain the following lemma. 
Lemma 3.3 Assume that the function $G(t, x)$ satisfies the following requirements: (i) $G(t, x)$ is measurable in $t \in E$ for every $x \in \mathbb{R}^{N}$ and continuous in $x$ for a.e. $t \in E$, where $0<$ meas $E<\infty$. (ii) There exists a constant $d>0$ such that $\lim _{|x| \rightarrow \infty} G(t, x)>d$, for a.e. $t \in E$. Then, for every $0<d_{0}<d$, and $\delta>0$, there exists a subset $E_{\delta} \subset E$ with meas $\left(E \backslash E_{\delta}\right)<\delta$ such that $\lim _{|x| \rightarrow \infty} G(t, x)>d_{0}$ uniformly for all $t \in E_{\delta}$.

Proof First, we show that the following conclusion is true.

Conclusion Assume that the function sequence $\left\{g_{n}\right\}$ satisfies the following: (i) $g_{n}(t)$ is measurable in $t \in E$. (ii) There exists constant $d>0$ such that $\lim _{n \rightarrow \infty} g_{n}(t)>d$, for a.e. $t \in E$. Then, for every $\delta>0$, there exists a subset $E_{\delta} \subset E$ with meas $\left(E \backslash E_{\delta}\right)<\delta$ such that $\lim _{n \rightarrow \infty} g_{n}(t)>d$ uniformly for all $t \in E_{\delta}$.

In fact, without loss of generality, we may assume that $\lim _{n \rightarrow \infty} g_{n}(t)>d$ for all $t \in E$. Define $E_{n}=\bigcap_{k=n}^{\infty}\left\{t \in E \mid g_{k}(t)>d\right\}$. Then $E_{n}$ is measurable and $E_{n} \subset E_{m}$ if $n<m$. Thus, $E=$ $\bigcup_{n=1}^{\infty} E_{n}$ and meas $E=\lim _{n \rightarrow \infty}$ meas $E_{n}$, which implies that $\lim _{n \rightarrow \infty}$ meas $\left(E \backslash E_{n}\right)=0$. Hence, for every $\delta>0$, there exists $n_{0}$ such that meas $\left(E \backslash E_{n_{0}}\right)<\delta$. Let $E_{\delta}=E_{n_{0}}$, then meas $(E \backslash$ $\left.E_{\delta}\right)<\delta$, and there exists $n_{0}$ such that $g_{n}(t)>d$ for all $t \in E_{\delta}$ as $n \geq n_{0}$, i.e. $\lim _{n \rightarrow \infty} g_{n}(t)>d$ uniformly for $t \in E_{\delta}$.

Now, let $g_{n}(t)=\inf _{|x| \geq n} G(t, x)$. Then by the continuity of $G(t, x)$ in $x$ for a.e. $t \in E$, we know that $g_{n}(t)$ is measurable for all $n$. Also, $\lim _{n \rightarrow \infty} g_{n}(t) \geq d$ for a.e. $t \in E$. Thus, for every $0<d_{0}<d$, and $\delta>0$, by the above conclusion, there exists a subset $E_{\delta} \subset E$ such that $\lim _{n \rightarrow \infty} g_{n}(t)>d_{0}$ uniformly for $t \in E_{\delta}$, and so, $\lim _{|x| \rightarrow \infty} G(t, x)>d_{0}$ uniformly for $t \in E_{\delta}$.

Lemma 3.4 Assume that $(\mathrm{H}),\left(\mathrm{H}_{1}\right)$, and $\left(\mathrm{H}_{2}\right)$ hold. Then $B(u) \geq 0$ for all $u \in E^{\alpha}$ and $B(u) \rightarrow \infty$ as $\|u\| \rightarrow \infty$ on any finite-dimensional subspace of $E^{\alpha}$.

Proof It is obvious that $B(u) \geq 0$ for all $u \in E^{\sigma}$ by $\left(\mathrm{H}_{1}\right)$ and (3.2).

Let $E$ be any finite-dimensional subspace of $E^{\alpha}$. We claim that $B(u) \rightarrow \infty$ as $\|u\| \rightarrow$ $\infty$ on $E$. In fact, for any $u \in E$, set $D_{u}=\left\{t \in[0, T]|| u(t) \mid \geq \varepsilon_{0}\|u\|\right\}$, where $\varepsilon_{0}$ is given in Lemma 3.2. Then meas $D_{u} \geq \varepsilon_{0}$ by Lemma 3.2. In terms of Lemma 3.3 combined with condition $\left(\mathrm{H}_{2}\right)$, we know that for $\delta=\varepsilon_{0} / 2$, and $0<d_{0}<d$, there exist $R>0$ and a subset $E_{\delta} \subset[0, T]$ with meas $\left([0, T] \backslash E_{\delta}\right)<\delta$ such that

$$
F(t, x)>d_{0}|x|^{\sigma} \quad \text { as }|x| \geq R \text { for all } t \in E_{\delta} .
$$

Now, for any $u \in E$, denote $E_{u}=D_{u} \cap E_{\delta}$, from

$$
\begin{aligned}
T-\operatorname{meas} E_{u} & =\operatorname{meas}\left([0, T] \backslash E_{u}\right) \\
& =\operatorname{meas}\left(\left([0, T] \backslash D_{u}\right) \cup\left([0, T] \backslash E_{\delta}\right)\right) \\
& \leq T-\operatorname{meas} D_{u}+\operatorname{meas}\left([0, T] \backslash E_{\delta}\right) \\
& <T-\varepsilon_{0} / 2,
\end{aligned}
$$

we get meas $E_{u}>\varepsilon_{0} / 2$. Thus, for any $u \in E$ with $\|u\| \geq \frac{R}{\varepsilon_{0}}$, by (3.9) we have

$$
F(t, u(t))>d_{0}|u(t)|^{\sigma} \geq d_{0} \varepsilon_{0}^{\sigma}\|u\|^{\sigma} \quad \text { for all } t \in E_{u},
$$


and therefore

$$
B(u)=\int_{0}^{T} F(t, u(t)) d t \geq \int_{E_{u}} F(t, u(t)) d t \geq \frac{1}{2} d_{0} \varepsilon_{0}^{\sigma+1}\|u\|^{\sigma} .
$$

This shows that $B(u) \rightarrow \infty$ as $\|u\| \rightarrow \infty$ on $E$. The proof is complete.

Lemma 3.5 Assume $(\mathrm{H}),\left(\mathrm{H}_{1}\right)$, and $\left(\mathrm{H}_{3}\right)$ hold. Then there exist $a k_{0}>0$ and two sequences $0<r_{k}<\rho_{k} \rightarrow 0$ as $k \rightarrow \infty$ such that for $k \geq k_{0}$, the following relations hold:

$$
\begin{aligned}
& a_{k}(\lambda)=\inf _{u \in Z_{k},\|u\|=\rho_{k}} \Phi_{\lambda}(u)>0, \quad b_{k}(\lambda)=\max _{u \in W_{k},\|u\|=r_{k}} \Phi_{\lambda}(u)<0, \quad \forall \lambda \in[1,2] ; \\
& d_{k}(\lambda)=\inf _{u \in Z_{k},\|u\| \leq \rho_{k}} \Phi_{\lambda}(u) \rightarrow 0 \quad \text { as } k \rightarrow \infty \text { uniformly for } \lambda \in[1,2] .
\end{aligned}
$$

Proof Let $\alpha_{k}=\sup _{u \in Z_{k},\|u\|=1}\|u\|_{2}$, we claim that $\alpha_{k} \rightarrow 0$ as $k \rightarrow \infty$. Indeed, if not, then there exist a constant $\varepsilon$ and a sequence $\left\{u_{j}\right\} \subset E^{\alpha}$ with $u_{j} \perp W_{k_{j-1}}$ such that $\left\|u_{j}\right\|=1$ and $\left\|u_{j}\right\|_{2} \geq \varepsilon$, where $k_{j} \geq 3, k_{j} \rightarrow \infty$ as $\rightarrow \infty$.

For any $v \in E^{\alpha}$, we may choose $w_{j} \in W_{k_{j}-1}$ such that $w_{j} \rightarrow v$ on $E^{\alpha}$ noting that $E^{\alpha}=$ $\overline{\bigoplus_{j=1}^{\infty} X_{j}}$. From

$$
\left|\left\langle u_{j}, v\right\rangle\right|=\left|\left\langle u_{j}, w_{j}-v\right\rangle\right| \leq\left\|u_{j}\right\|\left\|w_{j}-v\right\|=\left\|w_{j}-v\right\| \rightarrow 0 \quad \text { as } j \rightarrow \infty,
$$

we know that $u_{j} \rightarrow 0$ in $E^{\alpha}$, and therefore $u_{j} \rightarrow 0$ in $C[0, T]$ in view of Lemma 2.3. Consequently, $\left\|u_{j}\right\|_{2} \rightarrow 0$ as $j \rightarrow \infty$. This contradicts the fact that $\left\|u_{j}\right\|_{2} \geq \varepsilon$.

Now, take $\delta_{1}=\frac{\Gamma(\alpha)(2 \alpha-1)^{1 / 2}}{T^{\alpha-1 / 2}} \delta$, where $\delta$ is described in $\left(\mathrm{H}_{3}\right)$. Then for any $u \in E^{\alpha}$ with $\|u\| \leq$ $\delta_{1}$, it follows from Lemma 2.2 that $|u(t)| \leq \delta$, for any $t \in[0, T]$. Hence, according to $\left(\mathrm{H}_{3}\right)$, we get

$$
c(t)|u(t)|^{\tau} \leq F(t, u(t)) \leq l(t)|u(t)|^{\eta} \text {, a.e. } t \in[0, T]
$$

for any $u \in E^{\alpha}$ with $\|u\| \leq \delta_{1}$, and therefore

$$
\int_{0}^{T} c(t)|u(t)|^{\tau} d t \leq \int_{0}^{T} F(t, u(t)) d t \leq\|l(t)\|_{p}\|u(t)\|_{2}^{\eta}
$$

by the Hölder inequality. Thus, by Lemma 2.4 together with (3.1)-(3.2), we obtain

$$
\Phi_{\lambda}(u) \geq \frac{1}{2}|\cos (\alpha \pi)|\|u\|^{2}-2\|l\|_{p}\|u\|_{2}^{\eta}
$$

for any $u \in E^{\alpha}$ with $\|u\| \leq \delta_{1}$ and all $\lambda \in[1,2]$. In view of the definition of $\alpha_{k}$, we know that $\|u\|_{2} \leq \alpha_{k}\|u\|$ for any $u \in Z_{k}$. Then (3.11) implies that

$$
\Phi_{\lambda}(u) \geq \frac{1}{2}|\cos (\alpha \pi)|\|u\|^{2}-2\|l\|_{p} \alpha_{k}^{\eta}\|u\|^{\eta}
$$

for any $u \in Z_{k}$ with $\|u\| \leq \delta_{1}$. Set $c_{1}=\frac{1}{2}|\cos (\alpha \pi)|, c_{2}=2\|l\|_{p}$. Then (3.12) is reduced to the form

$$
\Phi_{\lambda}(u) \geq c_{1}\|u\|^{2}-c_{2} \alpha_{k}^{\eta}\|u\|^{\eta}
$$


for any $u \in Z_{k}$ with $\|u\| \leq \delta_{1}$. Let $\theta_{k}=\frac{2 c_{2}}{c_{1}} \alpha_{k}^{\eta}$. Obviously, $\theta_{k} \rightarrow 0$ as $k \rightarrow \infty$ since $\alpha_{k} \rightarrow 0$ as $k \rightarrow \infty$. Let $\rho_{k}=\theta_{k}^{\frac{1}{2-\eta}}$, then $\rho_{k} \rightarrow 0$ as $k \rightarrow \infty$, and therefore, there exists a $k_{0}>0$ such that $\rho_{k} \leq \delta_{1}$ as $k \geq k_{0}$. Hence, for any $k \geq k_{0}$ and $u \in Z_{k}$ with $\|u\|=\rho_{k}$, from (3.13), it follows that

$$
\Phi_{\lambda}(u) \geq c_{1} \rho_{k}^{2}-c_{2} \alpha_{k}^{\eta} \rho_{k}^{\eta}=\frac{c_{1}}{2} \rho_{k}^{2}>0 .
$$

So,

$$
a_{k}(\lambda)=\inf _{u \in Z_{k},\|u\|=\rho_{k}} \Phi_{\lambda}(u) \geq \frac{c_{1}}{2} \rho_{k}^{2}>0 .
$$

In addition, for any $k \geq k_{0}$, all $\lambda \in[1,2]$ and $u \in Z_{k}$ with $\|u\| \leq \rho_{k}$, noting that

$$
\Phi_{\lambda}(u) \geq-c_{2} \alpha_{k}^{\eta}\|u\|^{\eta} \geq-c_{2} \alpha_{k}^{\eta} \rho_{k}^{\eta} \rightarrow 0 \quad \text { as } k \rightarrow \infty
$$

we have

$$
d_{k}(\lambda)=\inf _{u \in Z_{k},\|u\| \leq \rho_{k}} \Phi_{\lambda}(u) \geq-c_{2} \alpha_{k}^{\eta} \rho_{k}^{\eta} \rightarrow 0 \quad \text { as } k \rightarrow \infty .
$$

This means that

$$
\lim _{k \rightarrow \infty} \inf d_{k}(\lambda) \geq 0
$$

Again, according to the fact that $F \geq 0$ on $[0, T] \times \mathbb{R}^{N}$, by applying Lemma 2.4, we obtain

$$
\begin{aligned}
\Phi_{\lambda}(u) & \leq-\frac{1}{2} \int_{0}^{T}\left({ }_{0}^{c} D_{t}^{\alpha} u(t),{ }_{t}^{c} D_{T}^{\alpha} u(t)\right) d t \\
& \leq \frac{1}{2|\cos (\alpha \pi)|}\|u\|^{2} \leq \frac{1}{2|\cos (\alpha \pi)|} \rho_{k}^{2}
\end{aligned}
$$

for any $u \in Z_{k}$ with $\|u\| \leq \rho_{k}$, and all $\lambda \in[1,2]$. Combining (3.15) with (3.16), we get $d_{k}(\lambda) \rightarrow 0$ as $k \rightarrow \infty$ uniformly for all $\lambda \in[1,2]$.

On the other hand, once again using (3.10), and applying the reverse Hölder inequality, for any $u \in E^{\alpha}$ with $\|u\| \leq \delta_{1}$, we have

$$
\begin{aligned}
\int_{0}^{T} F(t, u(t)) d t & \geq \int_{0}^{T} c(t)|u(t)|^{\tau} d \tau \\
& \geq\left(\int_{0}^{T} c(t)^{-r} d t\right)^{-1 / r}\left(\int_{0}^{T}|u(t)|^{s \tau} d t\right)^{1 / s}=c_{0}\|u\|_{s \tau}^{\tau}
\end{aligned}
$$

where $c_{0}=\left(\int_{0}^{T} c(t)^{-r} d t\right)^{-1 / r}, s=\frac{r}{1+r}$. In terms of the equivalence of any norm on finitedimensional space, we know that for any fixed $k \in \mathbb{N}$, there exists a constant $b_{k}>0$ such that $\|u\|_{s \tau} \geq b_{k}\|u\|$ for any $u \in W_{k}$. Thus, by Lemma 2.4, (3.1)-(3.2) and (3.17), we get

$$
\Phi_{\lambda}(u) \leq \frac{1}{2|\cos (\alpha \pi)|}\|u\|^{2}-c_{0} b_{k}^{\tau}\|u\|^{\tau}
$$


for any $u \in W_{k}$, and all $\lambda \in[1,2]$. Since $0<\tau<2$, we may choose $0<r_{k}<\min \left\{\delta_{1}, \rho_{k}\right\}$ small enough so that

$$
\frac{1}{2|\cos (\alpha \pi)|} r_{k}^{2}-c b_{k}^{\tau} r_{k}^{\tau}<0
$$

and therefore (3.18) implies

$$
b_{k}(\lambda)=\max _{u \in W_{k},\|u\|=r_{k}} \Phi_{\lambda}(u)<0
$$

for all $\lambda \in[1,2]$. The proof is complete.

Now, we are in a position to state our first result in the present paper.

Theorem 3.1 Assume that $(\mathrm{H}),\left(\mathrm{H}_{1}\right)-\left(\mathrm{H}_{4}\right)$ hold. Then BVP (1.2) has infinitely many small energy solutions $u_{k} \in E^{\alpha} \backslash\{0\}$ satisfying

$$
-\frac{1}{2} \int_{0}^{T}\left({ }_{0}^{c} D_{t}^{\alpha} u(t),{ }_{t}^{c} D_{T}^{\alpha} u(t)\right) d t-\int_{0}^{T} F(t, u(t)) d t \rightarrow 0^{-} \quad \text { as } k \rightarrow \infty .
$$

Proof By Lemma 2.2 and Lemma 2.4 combined with assumption (H), it is easy to see that $\Phi_{\lambda}$ maps bounded sets into bounded sets in $E^{\alpha}$ uniformly for $\lambda \in[1,2]$. Also, evidently, $\Phi_{\lambda}(u)=\Phi_{\lambda}(-u)$ for all $\lambda \in[1,2]$ and $u \in E^{\alpha}$ observing that condition $\left(\mathrm{H}_{1}\right)$ holds. Now, in terms of Lemma 2.5 together with Lemmas 3.4-3.5 and Remark 2.4, we conclude that, for every fixed $k \geq k_{0}$, there exists $\left\{\lambda_{n}^{k}\right\}$ with $\lambda_{n}^{k} \rightarrow 1$ as $n \rightarrow \infty$, and $u\left(\lambda_{n}^{k}\right) \in W_{n}$ such that

$$
\left.\Phi_{\lambda_{n}^{k}}^{\prime}\left(u\left(\lambda_{n}^{k}\right)\right)\right|_{W_{n}}=0, \quad \Phi_{\lambda_{n}}\left(u\left(\lambda_{n}^{k}\right)\right) \rightarrow c_{k} \in\left[d_{k}(2), b_{k}(1)\right] \quad \text { as } n \rightarrow \infty .
$$

We claim that the $\left\{u\left(\lambda_{n}^{k}\right)\right\}$ is bounded in $E^{\alpha}$. For simplicity, we still denote $\lambda_{n}^{k}$ as $\lambda_{n}$ in the following.

In fact, by Lemma 2.2 and Lemma 2.4 together with $\left(\mathrm{H}_{4}\right)$, we have

$$
\begin{aligned}
\left(\frac{1}{2}-\mu\right)|\cos (\alpha \pi)|\left\|u\left(\lambda_{n}\right)\right\|^{2} \\
\leq-\left(\frac{1}{2}-\mu\right) \int_{0}^{T}\left({ }_{0}^{c} D_{t}^{\alpha} u\left(\lambda_{n}\right)(t),{ }_{t}^{c} D_{T}^{\alpha} u\left(\lambda_{n}\right)(t)\right) d t \\
=\Phi_{\lambda_{n}}\left(u\left(\lambda_{n}\right)\right)-\mu\left\langle\Phi_{\lambda_{n}}^{\prime}\left(u\left(\lambda_{n}\right)\right), u\left(\lambda_{n}\right)\right\rangle \\
\quad+\lambda_{n} \int_{0}^{T}\left[F\left(t, u\left(\lambda_{n}\right)(t)\right)-\mu\left(\nabla F\left(t, u\left(\lambda_{n}\right)(t)\right), u\left(\lambda_{n}\right)(t)\right)\right] d t \\
\leq c_{k}+o(1)+2 \int_{0}^{T}\left|d(t) \| u\left(\lambda_{n}\right)(t)\right|^{\gamma} d t \\
\leq c_{k}+o(1)+2\|d\|_{2 /(2-\gamma)}\left\|u\left(\lambda_{n}\right)\right\|_{2}^{\gamma} \\
\leq c_{k}+o(1)+\frac{2\|d\|_{2 /(2-\gamma)} T^{\alpha \gamma}}{(\Gamma(\alpha+1))^{\gamma}}\left\|u\left(\lambda_{n}\right)\right\|^{\gamma},
\end{aligned}
$$

noting that $\Phi_{\lambda_{n}}\left(u\left(\lambda_{n}\right)\right) \rightarrow c_{k}$ as $n \rightarrow \infty$ and $\left.\Phi_{\lambda_{n}}^{\prime}\left(u\left(\lambda_{n}\right)\right)\right|_{W_{n}}=0$. Thus, it follows from (3.19) that the sequence $\left\{u\left(\lambda_{n}\right)\right\}$ is bounded. 
Now, we show that $\left\{u\left(\lambda_{n}\right)\right\}$ has a convergent subsequence for every fixed $k \geq k_{0}$.

In fact, owing to the fact that the sequence $\left\{u\left(\lambda_{n}\right)\right\}$ is bounded, and $E^{\alpha}$ is reflexive, we know that there exists $u \in E^{\alpha}$ such that, up to a subsequence, $u\left(\lambda_{n}\right) \rightarrow u$ in $E^{\alpha}$. Keeping in mind that $\left\{e_{j}\right\}$ is the completely orthonormal basis of $E^{\alpha}, W_{n}=L\left(e_{1}, e_{2}, \ldots, e_{n}\right)$, $u=\sum_{j=1}^{\infty}\left\langle e_{j}, u\right\rangle e_{j}$, and letting $P_{n}: E^{\alpha} \rightarrow W_{n}$ be the orthogonal projection operator, we know that $P_{n} u=\sum_{j=0}^{n}\left\langle e_{j}, u\right\rangle e_{j}$ and $P_{n} u \rightarrow u$ in $E^{\alpha}$ as $n \rightarrow \infty$. Therefore, $u\left(\lambda_{n}\right)-P_{n} u \rightarrow 0$ in $E^{\alpha}$ as $n \rightarrow \infty$. Thus $u\left(\lambda_{n}\right)-P_{n} u \rightarrow 0$ in $C[0, T]$ as $n \rightarrow \infty$. Also, by $u\left(\lambda_{n}\right)-P_{n} u \rightarrow 0$, there exists a constant $M_{1}>0$ such that $\left\|u\left(\lambda_{n}\right)-P_{n} u\right\| \leq M_{1}$ for all $n \in \mathbb{N}$. Moreover, from the fact that $\Phi_{1}^{\prime}(u) \in\left(E^{\alpha}\right)^{*}$, it follows that

$$
\left\langle\Phi_{1}^{\prime}(u), u\left(\lambda_{n}\right)-P_{n} u\right\rangle \rightarrow 0 \quad \text { as } n \rightarrow \infty .
$$

Also, since $\Phi_{1}^{\prime} \in C\left(E^{\alpha} \rightarrow\left(E^{\alpha}\right)^{*}\right)$, we have

$$
\begin{aligned}
\left|\left\langle\Phi_{1}^{\prime}\left(P_{n} u\right)-\Phi_{1}^{\prime}(u), u\left(\lambda_{n}\right)-P_{n} u\right\rangle\right| & \leq\left|\Phi_{1}^{\prime}\left(P_{n} u\right)-\Phi_{1}^{\prime}(u)\right|\left\|u\left(\lambda_{n}\right)-P_{n} u\right\| \\
& \leq M_{1}\left|\Phi_{1}^{\prime}\left(P_{n} u\right)-\Phi_{1}^{\prime}(u)\right| \rightarrow 0 \quad \text { as } n \rightarrow \infty,
\end{aligned}
$$

observing that $P_{n} u \rightarrow u$ in $E^{\alpha}$. Therefore, by (3.20), (3.21), we have

$$
\begin{aligned}
\left|\left\langle\Phi_{1}^{\prime}\left(P_{n} u\right), u\left(\lambda_{n}\right)-P_{n} u\right\rangle\right| \leq & \left|\left\langle\Phi_{1}^{\prime}\left(P_{n} u\right)-\Phi_{1}^{\prime}(u), u\left(\lambda_{n}\right)-P_{n} u\right\rangle\right| \\
& +\left|\left\langle\Phi_{1}^{\prime}(u), u\left(\lambda_{n}\right)-P_{n} u\right\rangle\right| \rightarrow 0
\end{aligned}
$$

as $n \rightarrow \infty$.

Now, by (3.22) and applying Lemma 2.4, we have

$$
\begin{aligned}
&|\cos (\alpha \pi)|\left\|P_{n}\left(u\left(\lambda_{n}\right)-u\right)\right\|^{2} \\
& \leq- \int_{0}^{T}\left({ }_{0}^{c} D_{t}^{\alpha} P_{n}\left(u\left(\lambda_{n}\right)-u\right)(t),{ }_{t}^{c} D_{T}^{\alpha} P_{n}\left(u\left(\lambda_{n}\right)-u\right)(t)\right) d t \\
&=\left\langle\Phi_{\lambda_{n}}^{\prime}\left(P_{n} u\left(\lambda_{n}\right)\right), P_{n}\left(u\left(\lambda_{n}\right)-u\right)\right\rangle-\left\langle\Phi_{1}^{\prime}\left(P_{n} u\right), P_{n}\left(u\left(\lambda_{n}\right)-u\right)\right\rangle \\
&+\lambda_{n} \int_{0}^{T}\left(\nabla F\left(t, P_{n} u\left(\lambda_{n}\right)\right), P_{n}\left(u\left(\lambda_{n}\right)-u\right)\right) d t-\int_{0}^{T}\left(\nabla F\left(t, P_{n} u\right), P_{n}\left(u\left(\lambda_{n}\right)-u\right)\right) d t \\
&=-\left\langle\Phi_{1}^{\prime}\left(P_{n} u\right), u\left(\lambda_{n}\right)-P_{n} u\right\rangle+\lambda_{n} \int_{0}^{T}\left(\nabla F\left(t, u\left(\lambda_{n}\right)(t)\right), u\left(\lambda_{n}\right)(t)-\left(P_{n} u\right)(t)\right) d t \\
& \quad-\int_{0}^{T}\left(\nabla F\left(t,\left(P_{n} u\right)(t)\right), u\left(\lambda_{n}\right)(t)-\left(P_{n} u\right)(t)\right) d t \\
& \leq\left|\left\langle\Phi_{1}^{\prime}\left(P_{n} u\right), u\left(\lambda_{n}\right)-P_{n} u\right\rangle\right|+2 \int_{0}^{T}\left|\nabla F\left(t, u\left(\lambda_{n}\right)(t)\right)\right|\left|u\left(\lambda_{n}\right)(t)-\left(P_{n} u\right)(t)\right| d t \\
&+\int_{0}^{T}\left|\nabla F\left(t,\left(P_{n} u\right)(t)\right)\right|\left|u\left(\lambda_{n}\right)(t)-\left(P_{n} u\right)(t)\right| d t:=Q_{n}
\end{aligned}
$$

noting that $P_{n} u\left(\lambda_{n}\right)=u\left(\lambda_{n}\right)$ since $u\left(\lambda_{n}\right) \in W_{n}$ and $\left\langle\Phi_{\lambda_{n}}^{\prime}\left(u\left(\lambda_{n}\right)\right), P_{n}\left(u\left(\lambda_{n}\right)-u\right)\right\rangle=0$ since $\left.\Phi_{\lambda_{n}}^{\prime}\left(u\left(\lambda_{n}\right)\right)\right|_{W_{n}}=0$. Also, according to $u\left(\lambda_{n}\right) \rightarrow u, P_{n} u \rightarrow u$ in $E^{\alpha}$ as $n \rightarrow \infty$, there exists $M_{2}>0$ such that $\left\|u\left(\lambda_{n}\right)\right\| \leq M_{2},\left\|P_{n} u\right\| \leq M_{2}$ for all $n \in \mathbb{N}$. Therefore, it follows from 
Lemma 2.2 that $\left|u\left(\lambda_{n}\right)\right|_{\infty} \leq M_{3},\left|P_{n} u\right|_{\infty} \leq M_{3}$ for all $n \in \mathbb{N}$ for some $M_{3}>0$. Then, by (H), we immediately obtain

$$
\left|\nabla F\left(t, u\left(\lambda_{n}\right)(t)\right)\right| \leq \max _{|x| \leq M_{3}} a(|x|) b(t), \quad\left|\nabla F\left(t,\left(P_{n} u\right)(t)\right)\right| \leq \max _{|x| \leq M_{3}} a(|x|) b(t)
$$

for all $n \in \mathbb{N}$. Thus,

$$
\begin{aligned}
& \int_{0}^{T}\left|\nabla F\left(t, u\left(\lambda_{n}\right)(t)\right)\right|\left|u\left(\lambda_{n}\right)(t)-\left(P_{n} u\right)(t)\right| d t \\
& \quad \leq \max _{|x| \leq M_{3}} a(|x|)\left|u\left(\lambda_{n}\right)-P_{n} u\right|_{\infty} \int_{0}^{T} b(t) d t \rightarrow 0
\end{aligned}
$$

as $n \rightarrow \infty$, and

$$
\begin{aligned}
& \int_{0}^{T}\left|\nabla F\left(t,\left(P_{n} u\right)(t)\right)\right|\left|u\left(\lambda_{n}\right)(t)-\left(P_{n} u\right)(t)\right| d t \\
& \quad \leq \max _{|x| \leq M_{3}} a(|x|)\left|u\left(\lambda_{n}\right)-P_{n} u\right|_{\infty} \int_{0}^{T} b(t) d t \rightarrow 0
\end{aligned}
$$

as $n \rightarrow \infty$, since $u\left(\lambda_{n}\right)-P_{n} u \rightarrow 0$ in $C[0, T]$ as before. Thus, by (3.23) we know that $Q_{n} \rightarrow$ 0 as $n \rightarrow \infty$ according to (3.22). Also, by (3.23), we have

$$
\left\|u\left(\lambda_{n}\right)-P_{n} u\right\|=\left\|P_{n}\left(u\left(\lambda_{n}\right)-u\right)\right\| \leq\left(\frac{\left\|Q_{n}\right\|}{|\cos (\alpha \pi)|}\right)^{1 / 2}
$$

for all $n \in \mathbb{N}$. On the other hand, since $P_{n} u-u \rightarrow 0$ in $E^{\alpha}$ as $n \rightarrow \infty$, for arbitrary $\varepsilon>0$, there exists a $N>0$ such that $\left\|P_{n} u-u\right\|<\varepsilon$ as $n>N$. Thus

$$
\left\|u\left(\lambda_{n}\right)-P_{n} u\right\| \geq\left\|u\left(\lambda_{n}\right)-u\right\|-\left\|u-P_{n} u\right\| \geq\left\|u\left(\lambda_{n}\right)-u\right\|-\varepsilon
$$

as $n \geq N$. Consequently, by (3.24) and (3.25), we have

$$
\left\|u\left(\lambda_{n}\right)-u\right\| \leq\left(\frac{\left\|Q_{n}\right\|}{|\cos (\alpha \pi)|}\right)^{1 / 2}+\varepsilon
$$

as $n \geq N$, and therefore,

$$
\varlimsup_{n \rightarrow \infty}\left\|u\left(\lambda_{n}\right)-u\right\| \leq \lim _{n \rightarrow \infty}\left(\frac{\left\|Q_{n}\right\|}{|\cos (\alpha \pi)|}\right)^{1 / 2}+\varepsilon=\varepsilon,
$$

which means that $\varlimsup_{n \rightarrow \infty}\left\|u\left(\lambda_{n}\right)-u\right\|=0$ because of the arbitrariness of $\varepsilon$; namely, $u\left(\lambda_{n}\right) \rightarrow u$ in $E^{\alpha}$. Hence, by Lemma 2.5 and Remark 2.4, we know that Theorem 3.1 is true. This completes the proof.

Now, we establish another result in this paper. To this end, we first give some assumptions.

$\left(\mathrm{H}_{2}\right)^{\prime}$ There exist constants $c>0, p>2$, and $0<q \leq p$ such that

$$
F(t, x) \leq c\left(|x|^{p}+|x|^{q}\right) \quad \text { for all } x \in \mathbb{R}^{N} \text { and a.e. } t \in[0, T] .
$$


$\left(\mathrm{H}_{3}\right)^{\prime} \lim _{|x| \rightarrow 0} \sup \frac{F(t, x)}{|x|^{2}}<\frac{(\Gamma(\alpha+1))^{2}}{4 T^{2 \alpha}}|\cos (\alpha \pi)|$ uniformly for a.e. $t \in[0, T]$.

$\left(\mathrm{H}_{4}\right)^{\prime} \lim _{|x| \rightarrow \infty} \frac{F(t, x)}{|x|^{2}}=\infty$ for a.e. $t \in[0, T]$.

$\left(\mathrm{H}_{5}\right)^{\prime}$ There exist constants $\mu>2$ and $d>0$ such that

$$
\mu F(t, x)-(\nabla F(t, x), x) \leq d\left(1+|x|^{2}\right) \quad \text { for all } x \in \mathbb{R} \text {, and a.e. } t \in[0, T]
$$

By Lemma 3 in [26], we can easily see that the following lemma is true.

Lemma 3.6 Assume that $(\mathrm{H}),\left(\mathrm{H}_{4}\right)^{\prime}$ hold. Then for every $\delta>0$ there exists a subset $E_{\delta} \subset$ $[0, T]$ with meas $\left([0, T] \backslash E_{\delta}\right)<\delta$ such that

$$
\lim _{|x| \rightarrow \infty} \frac{F(t, x)}{|x|^{2}}=\infty \quad \text { uniformly for } t \in E_{\delta} .
$$

We establish the following lemma to obtain the second result in this paper.

Lemma 3.7 Let $(\mathrm{H}),\left(\mathrm{H}_{1}\right),\left(\mathrm{H}_{2}\right)^{\prime}-\left(\mathrm{H}_{4}\right)^{\prime}$ be satisfied. Then there exist two sequences $\rho_{k}>r_{k}>$ 0 such that

$$
b_{k}=\inf _{u \in Z_{k},\|u\|=r_{k}} \Phi_{\lambda}(u)>a_{k}(\lambda)=\max _{u \in W_{k},\|u\|=\rho_{k}} \Phi_{\lambda}(u), \quad \forall \lambda \in[1,2] .
$$

Proof We divide the proof into two parts.

Part I. In this part, we will prove that there exists a sequence $r_{k}>0$ such that

$$
b_{k}=\inf _{u \in Z_{k},\|u\|=r_{k}} \Phi_{\lambda}(u)>0, \quad \forall \lambda \in[1,2] .
$$

In fact, by $\left(\mathrm{H}_{3}\right)^{\prime}$, there exists a subset $E_{0} \subset[0, T]$ with meas $E_{0}=T$ such that $d_{0}<$ $\frac{(\Gamma(\alpha+1))^{2}}{4 T^{2 \alpha}}|\cos (\alpha \pi)|$, where $d_{0}=\sup _{t \in E_{0}}\left(\lim _{|x| \rightarrow 0} \sup \frac{F(t, x)}{|x|^{2}}\right)$. Taking $\varepsilon>0$ such that $d_{0}<$ $\frac{(\Gamma(\alpha+1))^{2}}{4 T^{2 \alpha}}|\cos (\alpha \pi)|-\varepsilon / 2$, it follows that there exists $\delta>0$ such that

$$
|F(t, x)|<\left(\frac{(\Gamma(\alpha+1))^{2}}{4 T^{2 \alpha}}|\cos (\alpha \pi)|-\varepsilon / 2\right)|x|^{2}
$$

as $|x| \leq \delta$ for $t \in E_{0}$. By $\left(\mathrm{H}_{2}\right)^{\prime}$ together with (3.26), we know that there exists a constant $c_{\varepsilon}>0$ such that

$$
|F(t, x)| \leq\left(\frac{(\Gamma(\alpha+1))^{2}}{4 T^{2 \alpha}}|\cos (\alpha \pi)|-\varepsilon / 2\right)|x|^{2}+c_{\varepsilon}|x|^{p}
$$

for all $x \in \mathbb{R}^{N}$ and a.e. $t \in[0, T]$.

Let $\alpha_{k}=\sup _{u \in Z_{k},\|u\|=1}\|u\|_{p}$. Using the method as in the proof of Lemma 3.5, we can deduce that $\alpha_{k} \rightarrow 0$ as $k \rightarrow \infty$. Thus for any $u \in Z_{k}$, by (3.1), (3.27), Lemma 2.2, and Lemma 2.4, we have

$$
\begin{aligned}
\Phi_{\lambda}(u) & \geq \frac{1}{2}|\cos (\alpha \pi)|\|u\|^{2}-2 \int_{0}^{T} F(t, u(t)) d t \\
& \geq \frac{1}{2}|\cos (\alpha \pi)|\|u\|^{2}-2\left(\frac{(\Gamma(\alpha+1))^{2}}{4 T^{2 \alpha}}|\cos (\alpha \pi)|-\frac{\varepsilon}{2}\right) \int_{0}^{T}|u(t)|^{2} d t
\end{aligned}
$$




$$
\begin{aligned}
& -2 c_{\varepsilon} \int_{0}^{T}|u(t)|^{p} d t \\
\geq & \frac{1}{2}|\cos (\alpha \pi)|\|u\|^{2}-\left(\frac{1}{2}|\cos (\alpha \pi)|-\frac{\varepsilon T^{2 \alpha}}{(\Gamma(\alpha+1))^{2}}\right)\|u\|^{2}-2 c_{\varepsilon} \alpha_{k}^{p}\|u\|^{p} \\
= & \frac{\varepsilon T^{2 \alpha}}{(\Gamma(\alpha+1))^{2}}\|u\|^{2}-2 c_{\varepsilon} \alpha_{k}^{p}\|u\|^{p} .
\end{aligned}
$$

According to $p>2$ and $\alpha_{k} \rightarrow 0$ as $k \rightarrow \infty$, if we take $r_{k}=\left(\frac{\varepsilon T^{2 \alpha}}{4 c_{\varepsilon}(\Gamma(\alpha+1))^{2}}\right)^{\frac{1}{p-2}} \alpha_{k}^{\frac{p}{2-p}}$, then $r_{k} \rightarrow \infty$ as $k \rightarrow \infty$, and

$$
\Phi_{\lambda}(u) \geq c_{\varepsilon}^{\frac{2}{2-p}}\left(\frac{\varepsilon T^{2 \alpha}}{2(\Gamma(\alpha+1))^{2}}\right)^{\frac{p}{p-2}} \alpha_{k}^{\frac{2 p}{2-p}}>0, \quad \forall \lambda \in[1,2]
$$

for any $u \in Z_{k}$ with $\|u\|=r_{k}$. Thus,

$$
b_{k}(\lambda)=\inf _{u \in Z_{k},\|u\|=r_{k}} \Phi_{\lambda}(u) \geq c_{\varepsilon}^{\frac{2}{2-p}}\left(\frac{\varepsilon T^{2 \alpha}}{2(\Gamma(\alpha+1))^{2}}\right)^{\frac{p}{p-2}} \alpha_{k}^{\frac{2 p}{2-p}}>0, \quad \forall \lambda \in[1,2] .
$$

Part II. In this part, we will show that there exists a sequence $\left\{\rho_{k}\right\}$ with $\rho_{k}>r_{k}$ such that

$$
a_{k}(\lambda)=\max _{u \in W_{k},\|u\|=\rho_{k}} \Phi_{\lambda}(u)<0, \quad \forall \lambda \in[1,2] .
$$

In fact, for any fixed $k \in \mathbb{N}$, by Lemma 3.2, we know that there exists a constant $\varepsilon_{k}>0$ such that

$$
\operatorname{meas}\left\{t \in[0, T]:|u(t)| \geq \varepsilon_{k}\|u\|\right\} \geq \varepsilon_{k}
$$

for any $u \in W_{k}$. By Lemma 3.6, for $0<\delta_{k}<\varepsilon_{k} / 2$, there exists $E_{k} \subset[0, T]$ with meas $([0, T] \backslash$ $\left.E_{k}\right)<\delta_{k}$ such that

$$
\lim _{|x| \rightarrow \infty} \frac{F(t, x)}{|x|^{2}}=\infty \quad \text { uniformly for } t \in E_{k} .
$$

Thus, for $M_{k}=\frac{2}{|\cos (\alpha \pi)| \varepsilon_{k}^{3}}$, it follows that there exists $R_{k}>0$ such that

$$
F(t, x)>M_{k}|x|^{2} \quad \text { for all } t \in E_{k}
$$

as $|x| \geq R_{k}$. Take $S_{k}=R_{k} / \varepsilon_{k}$ and let $D_{u}=\left\{t \in[0, T]\left\|u(t) \mid \geq \varepsilon_{k}\right\| u \|\right\}$. Then, for any $u \in W_{k}$ with $\|u\| \geq S_{k}$, we have

$$
u(t) \geq \varepsilon_{k}\|u\| \geq R_{k} \quad \text { for } t \in D_{u} .
$$

Let $E_{u}=D_{u} \cap E_{k}$. Then it follows from (3.29)-(3.30) that

$$
F(t, u(t))>M_{k}|u(t)|^{2} \quad \text { for all } t \in E_{u}
$$

for any $u \in W_{k}$ with $\|u\| \geq S_{k}$. 
Since

$$
\begin{aligned}
T-\operatorname{meas} E_{u} & =\operatorname{meas}\left([0, T] \backslash E_{u}\right) \\
& =\operatorname{meas}\left(\left([0, T] \backslash D_{u}\right) \cup\left([0, T] \backslash E_{k}\right)\right) \\
& \leq T-\operatorname{meas} D_{u}+\operatorname{meas}\left([0, T] \backslash E_{k}\right) \\
& <T-\varepsilon_{k} / 2,
\end{aligned}
$$

we know that meas $E_{u}>\varepsilon_{k} / 2$.

Thus, by (3.28), (3.30)-(3.31), and Lemma 2.4, for any $u \in W_{k}$ with $\|u\| \geq S_{k}$, we have

$$
\begin{aligned}
\Phi_{\lambda}(u) & \leq \frac{1}{2|\cos (\alpha \pi)|}\|u\|^{2}-\int_{0}^{T} F(t, u(t)) d t \\
& \leq \frac{1}{2|\cos (\alpha \pi)|}\|u\|^{2}-\int_{E_{u}} F(t, u(t)) d t \\
& \leq \frac{1}{2|\cos (\alpha \pi)|}\|u\|^{2}-M_{k} \int_{E_{u}}|u(t)|^{2} d t \\
& \leq \frac{1}{2|\cos (\alpha \pi)|}\|u\|^{2}-\frac{1}{2} M_{k} \varepsilon_{k}^{3}\|u\|^{2} \\
& =-\frac{1}{2|\cos (\alpha \pi)|}\|u\|^{2} .
\end{aligned}
$$

So, choosing $\rho_{k}>\max \left\{S_{k}, r_{k}\right\}$, it follows from (3.32) that

$$
\Phi_{\lambda}(u) \leq-\frac{1}{2|\cos (\alpha \pi)|} \rho_{k}^{2}
$$

for any $u \in W_{k}$ with $\|u\|=\rho_{k}$. Hence

$$
a_{k}(\lambda)=\max _{u \in W_{k},\|u\|=\rho_{k}} \Phi_{\lambda}(u)<0, \quad \forall \lambda \in[1,2] \text {, and } \rho_{k}>r_{k}
$$

Consequently, from Part I and Part II we conclude that Lemma 3.7 is true.

Now, we state another result in this paper.

Theorem 3.2 Assume that $(\mathrm{H}),\left(\mathrm{H}_{1}\right),\left(\mathrm{H}_{2}\right)^{\prime}-\left(\mathrm{H}_{5}\right)^{\prime}$ hold. Then BVP (1.1) has infinitely many high energy solutions $u_{k} \in E^{\alpha} \backslash\{0\}$ satisfying

$$
-\frac{1}{2} \int_{0}^{T}\left({ }_{0}^{c} D_{t}^{\alpha} u_{k}(t),{ }_{t}^{c} D_{T}^{\alpha} u_{k}(t)\right) d t-\int_{0}^{T} F\left(t, u_{k}(t)\right) d t \rightarrow \infty \quad \text { as } k \rightarrow \infty .
$$

Proof As in the proof of Theorem 3.1, it is easy to see that the conditions $\left(\mathrm{B}_{1}\right),\left(\mathrm{B}_{2}\right)$, and $\left(\mathrm{B}_{4}\right)$ of Lemma 2.6 hold under the assumptions $(\mathrm{H})$ and $\left(\mathrm{H}_{1}\right)$ combined with the fact that $A(u) \rightarrow \infty$ as $\|u\| \rightarrow \infty$, and applying Lemma 3.7. Consequently, by Lemma 2.6, we know that for a.e. $\lambda \in[1,2]$, there exists a sequence $\left\{u_{n}^{k}(\lambda)\right\}_{n=1}^{\infty}$ such that

$$
\sup _{n}\left\|u_{n}^{k}(\lambda)\right\|<\infty, \quad \Phi_{\lambda}^{\prime}\left(u_{n}^{k}(\lambda)\right) \rightarrow 0 \quad \text { and } \quad \Phi_{\lambda}\left(u_{n}^{k}(\lambda)\right) \rightarrow c_{k}(\lambda)
$$

as $n \rightarrow \infty$. 
Owing to the fact that $p>2$, it follows that

$$
c_{k}(\lambda) \geq b_{k}(\lambda) \geq c_{\varepsilon}^{\frac{2}{2-p}}\left(\frac{\varepsilon T^{2 \alpha}}{2(\Gamma(\alpha+1))^{2}}\right)^{\frac{p}{p-2}} \alpha_{k}^{\frac{2 p}{2-p}}:=\bar{b}_{k} \rightarrow \infty
$$

as $k \rightarrow \infty$ because $\alpha_{k} \rightarrow 0$ as $k \rightarrow \infty$. Also since

$$
c_{k}(\lambda)=\inf _{\gamma \in \Gamma_{k}} \max _{u \in B_{k}} \Phi_{\lambda}(\gamma(u)) \leq \max _{u \in B_{k}} \Phi_{\lambda}(u) \leq \max _{u \in B_{k}} \Phi_{1}(u):=\bar{c}_{k}
$$

we have

$$
\bar{b}_{k} \leq c_{k}(\lambda) \leq \bar{c}_{k} \quad \text { for all } \lambda \in[1,2]
$$

We choose a sequence $\lambda_{m} \rightarrow 1$ as $m \rightarrow \infty$ with $\lambda_{m} \in$ [1,2] such that (3.33) holds for all $\lambda_{m}(m=1,2, \ldots)$. Using arguments similar to the proof of Theorem 3.1, we can prove that the sequence $\left\{u_{n}^{k}\left(\lambda_{m}\right)\right\}_{n=1}^{\infty}$ has a strongly convergent subsequence. Now, we show this simply as follows.

In fact, since $\left\{u_{n}^{k}\left(\lambda_{m}\right)\right\}_{n=1}^{\infty}$ is bounded, there exists a $u^{k}\left(\lambda_{m}\right) \in E^{\alpha}$, up to a subsequence, such that $u_{n}^{k}\left(\lambda_{m}\right) \rightarrow u^{k}\left(\lambda_{m}\right)$ as $n \rightarrow \infty$, and therefore, $u_{n}^{k}\left(\lambda_{m}\right) \rightarrow u^{k}\left(\lambda_{m}\right)$ in $C[0, T]$ as $n \rightarrow$ $\infty$, keeping in mind Lemma 2.3. Let $M>0$ be a constant such that $\left\|u_{n}^{k}\left(\lambda_{m}\right)-u^{k}\left(\lambda_{m}\right)\right\| \leq M$, $\left\|u_{n}^{k}\left(\lambda_{m}\right)\right\| \leq M, \forall n \geq 1$. Then there exists $M_{1}$ such that $\left|u_{n}^{k}\left(\lambda_{m}\right)(t)\right| \leq M_{1}, \forall n \geq 1$, for all $t \in[0, T],\left|u^{k}\left(\lambda_{m}\right)(t)\right| \leq M_{1}$, for all $t \in[0, T]$ by Lemma 2.2. Moreover, by $u_{n}^{k}\left(\lambda_{m}\right) \rightarrow u^{k}\left(\lambda_{m}\right)$, and $\Phi_{1}^{\prime}\left(u^{k}\left(\lambda_{m}\right)\right) \in\left(E^{\alpha}\right)^{*}$, we know that $\left\langle\Phi_{1}^{\prime}\left(u^{k}\left(\lambda_{m}\right)\right), u_{n}^{k}\left(\lambda_{m}\right)-u^{k}\left(\lambda_{m}\right)\right\rangle \rightarrow 0$ as $n \rightarrow \infty$. Also from $\Phi_{\lambda_{m}}^{\prime}\left(u_{n}^{k}\left(\lambda_{m}\right)\right) \rightarrow 0$ as $n \rightarrow \infty$ and $\left\|u_{n}^{k}\left(\lambda_{m}\right)-u^{k}\left(\lambda_{m}\right)\right\| \leq M$, it follows that $\left\langle\Phi_{\lambda_{m}}^{\prime}\left(u_{n}^{k}\left(\lambda_{m}\right)\right), u_{n}^{k}\left(\lambda_{m}\right)-u^{k}\left(\lambda_{m}\right)\right\rangle \rightarrow 0$ as $n \rightarrow \infty$. Hence, by Lemma 2.4 and conditions $(\mathrm{H})$ and $\left(\mathrm{H}_{1}\right)$, we get

$$
\begin{aligned}
|\cos (\alpha \pi)|\left\|u_{n}^{k}\left(\lambda_{m}\right)-u^{k}\left(\lambda_{m}\right)\right\|^{2} & \\
\leq & -\int_{0}^{T}\left({ }_{0}^{c} D_{t}^{\alpha}\left(u_{n}^{k}\left(\lambda_{m}\right)-u^{k}\left(\lambda_{m}\right)\right),{ }_{t}^{c} D_{T}^{\alpha}\left(u_{n}^{k}\left(\lambda_{m}\right)-u^{k}\left(\lambda_{m}\right)\right)\right) d t \\
=\langle & \left.\Phi_{\lambda_{m}}^{\prime}\left(u_{n}^{k}\left(\lambda_{m}\right)\right), u_{n}^{k}\left(\lambda_{m}\right)-u^{k}\left(\lambda_{m}\right)\right\rangle-\left\langle\Phi_{1}^{\prime}\left(u^{k}\left(\lambda_{m}\right)\right), u_{n}^{k}\left(\lambda_{m}\right)-u^{k}\left(\lambda_{m}\right)\right\rangle \\
& +\lambda_{m} \int_{0}^{T}\left(\nabla F\left(t, u_{n}^{k}\left(\lambda_{m}\right)(t)\right), u_{n}^{k}\left(\lambda_{m}\right)(t)-u^{k}\left(\lambda_{m}\right)(t)\right) d t \\
& \quad-\int_{0}^{T}\left(\nabla F\left(t, u^{k}\left(\lambda_{m}\right)(t)\right), u_{n}^{k}\left(\lambda_{m}\right)(t)-u^{k}\left(\lambda_{m}\right)(t)\right) d t \\
\leq & o(1)+2\left|u_{n}^{k}\left(\lambda_{m}\right)-u^{k}\left(\lambda_{m}\right)\right|_{\infty} \int_{0}^{T}\left|\nabla F\left(t, u_{n}^{k}\left(\lambda_{m}\right)(t)\right)\right| d t \\
& +\left|u_{n}^{k}\left(\lambda_{m}\right)-u^{k}\left(\lambda_{m}\right)\right|_{\infty} \int_{0}^{T}\left|\nabla F\left(t, u^{k}\left(\lambda_{m}\right)(t)\right)\right| d t \\
\leq & o(1)+3\left|u_{n}^{k}\left(\lambda_{m}\right)-u^{k}\left(\lambda_{m}\right)\right|_{\infty} \max _{|x| \leq M_{1}} a(|x|) \int_{0}^{T} b(t) d t \rightarrow 0
\end{aligned}
$$

as $n \rightarrow \infty$. Thus, we conclude that $u_{n}^{k}\left(\lambda_{m}\right) \rightarrow u^{k}\left(\lambda_{m}\right)$ as $n \rightarrow \infty$ in $E^{\alpha}$. Hence, it follows from (3.33)-(3.34) that

$$
\Phi_{\lambda_{m}}^{\prime}\left(u^{k}\left(\lambda_{m}\right)\right)=0 \quad \text { and } \quad \Phi_{\lambda_{m}}\left(u^{k}\left(\lambda_{m}\right)\right) \in\left[\bar{b}_{k}, \bar{c}_{k}\right]
$$


We claim that the sequence $\left\{u^{k}\left(\lambda_{m}\right)\right\}_{m=1}^{\infty}$ is bounded.

In fact, if not, then there exists a subsequence of $\left\{u^{k}\left(\lambda_{m}\right)\right\}_{m=1}^{\infty}$, still denoted by $\left\{u^{k}\left(\lambda_{m}\right)\right\}_{m=1}^{\infty}$, such that $\left\|u^{k}\left(\lambda_{m}\right)\right\| \rightarrow \infty$ as $m \rightarrow \infty$. Let $v_{m}=\frac{u^{k}\left(\lambda_{m}\right)}{\left\|u^{k}\left(\lambda_{m}\right)\right\|}$. Then there exists a $v \in E^{\alpha}$, up to a subsequence, such that $v_{m} \rightarrow v$ in $E^{\alpha}$, and therefore $v_{m} \rightarrow v$ in $C[0, T]$ by Lemma 2.3.

Case 1. If $v \neq 0$ in $E^{\alpha}$, then meas $E_{0}>0$, where $E_{0}=\{t \in[0, T] \mid v(t) \neq 0\}$. By Lemma 2.4, condition $\left(\mathrm{H}_{1}\right)$, and the fact that $\Phi_{\lambda_{m}}\left(u^{k}\left(\lambda_{m}\right)\right) \in\left[\bar{b}_{k}, \bar{c}_{k}\right]$, we have

$$
\begin{aligned}
& \frac{1}{2|\cos (\alpha \pi)|}+o(1) \\
& =\frac{1}{2|\cos (\alpha \pi)|}+\frac{\left|\Phi_{\lambda_{m}}\left(u^{k}\left(\lambda_{m}\right)\right)\right|}{\left\|u^{k}\left(\lambda_{m}\right)\right\|^{2}} \\
& \geq-\frac{1}{2\left\|u^{k}\left(\lambda_{m}\right)\right\|^{2}} \int_{0}^{T}\left({ }_{0}^{c} D_{t}^{\alpha} u^{k}\left(\lambda_{m}\right)(t),{ }_{t}^{c} D_{T}^{\alpha} u^{k}\left(\lambda_{m}\right)(t)\right) d t-\frac{\Phi_{\lambda_{m}}\left(u^{k}\left(\lambda_{m}\right)\right)}{\left\|u^{k}\left(\lambda_{m}\right)\right\|^{2}} \\
& =\lambda_{m} \int_{0}^{T} \frac{F\left(t, u^{k}\left(\lambda_{m}\right)(t)\right)}{\left\|u^{k}\left(\lambda_{m}\right)\right\|^{2}} d t \\
& \geq \int_{E_{0}} \frac{F\left(t, u^{k}\left(\lambda_{m}\right)(t)\right)}{\left\|u^{k}\left(\lambda_{m}\right)\right\|^{2}} d t \\
& =\int_{E_{0}}\left|v_{m}(t)\right|^{2} \frac{F\left(t, u^{k}\left(\lambda_{m}\right)(t)\right)}{\left|u^{k}\left(\lambda_{m}\right)\right|^{2}} d t .
\end{aligned}
$$

Since $\left|u^{k}\left(\lambda_{m}\right)(t)\right|=\left|v_{m}(t)\right|\left\|u^{k}\left(\lambda_{m}\right)\right\| \rightarrow \infty$ as $m \rightarrow \infty$ for all $t \in E_{0}$, by $\left(\mathrm{H}_{4}\right)^{\prime}$ we see that

$$
\frac{F\left(t, u^{k}\left(\lambda_{m}\right)(t)\right)}{\left|u^{k}\left(\lambda_{m}\right)(t)\right|^{2}}\left|v_{m}(t)\right|^{2} \rightarrow \infty \quad \text { for a.e. } t \in E_{0} .
$$

Thus, using Fatou's result, we obtain $\underline{\lim }_{m \rightarrow \infty} \int_{E_{0}}\left|v_{m}(t)\right|^{2} \frac{F\left(t, u^{k}\left(\lambda_{m}\right)(t)\right)}{\left|u^{k}\left(\lambda_{m}\right)(t)\right|^{2}} d t=\infty$, which contradicts (3.35).

Case 2. If $v=0$ in $E^{\alpha}$, then $v(t)=0$ for all $t \in[0, T]$ by Lemma 2.2. Thus, by condition $\left(\mathrm{H}_{5}\right)^{\prime}$ together with Lemma 2.4, we have

$$
\begin{aligned}
\left(\frac{\mu}{2}-1\right)|\cos (\alpha \pi)| \\
\leq\left(1-\frac{\mu}{2}\right) \frac{1}{\left\|u^{k}\left(\lambda_{m}\right)\right\|^{2}} \int_{0}^{T}\left({ }_{0}^{c} D_{t}^{\alpha} u^{k}\left(\lambda_{m}\right)(t),{ }_{t}^{c} D_{T}^{\alpha} u^{k}\left(\lambda_{m}\right)(t)\right) d t \\
=\frac{1}{\left\|u^{k}\left(\lambda_{m}\right)\right\|^{2}}\left[\mu \Phi_{\lambda_{m}}\left(u^{k}\left(\lambda_{m}\right)\right)-\left\langle\Phi_{\lambda_{m}}^{\prime}\left(u^{k}\left(\lambda_{m}\right)\right), u^{k}\left(\lambda_{m}\right)\right\rangle\right] \\
\quad+\frac{\lambda_{m}}{\left\|u^{k}\left(\lambda_{m}\right)\right\|^{2}} \int_{0}^{T}\left(\mu F\left(t, u^{k}\left(\lambda_{m}\right)(t)\right)-\nabla F\left(t, u^{k}\left(\lambda_{m}\right)(t)\right), u^{k}\left(\lambda_{m}\right)(t)\right) d t \\
\leq o(1)+\frac{2 d}{\left\|u^{k}\left(\lambda_{m}\right)\right\|^{2}} \int_{0}^{T}\left(1+\left|u^{k}\left(\lambda_{m}\right)(t)\right|^{2}\right) d t \\
=o(1)+\frac{2 d T}{\left\|u^{k}\left(\lambda_{m}\right)\right\|^{2}}+2 d \int_{0}^{T}\left|v_{m}(t)\right|^{2} d t,
\end{aligned}
$$

noting that $\Phi_{\lambda_{m}}^{\prime}\left(u^{k}\left(\lambda_{m}\right)\right)=0$ and $\Phi_{\lambda_{m}}\left(u^{k}\left(\lambda_{m}\right)\right) \in\left[\bar{b}_{k}, \bar{c}_{k}\right]$. Thus letting $m \rightarrow \infty$ in (3.36), we obtain $\left(\frac{\mu}{2}-1\right)|\cos (\alpha \pi)| \leq 0$, which contradicts the fact that $\mu>2$. 
So, summing up the above analysis on Case 1 and Case 2, we know that the sequence $\left\{u^{k}\left(\lambda_{m}\right)\right\}_{m=1}^{\infty}$ is bounded in $E^{\alpha}$. Hence, there exists a $u^{k} \in E^{\alpha}$, up to a subsequence, such that $u^{k}\left(\lambda_{m}\right) \rightarrow u^{k}$ in $E^{\alpha}$ as $m \rightarrow \infty$, and therefore, $u^{k}\left(\lambda_{m}\right) \rightarrow u^{k}$ in $C[0, T]$ as $m \rightarrow \infty$. By the standard method, we can prove that $u^{k}$ is a critical point of $\Phi_{1}$. Taking into account the fact that $\Phi_{1}\left(u^{k}\right) \geq \bar{b}_{k} \rightarrow \infty$ as $k \rightarrow \infty$, we know that BVP (1.2), i.e., BVP (1.1) has infinitely many high energy solutions. The proof is complete.

Let us draw a conclusion for this paper. Because of applying the variant fountain theorems, the results for the existence of infinitely many small or high energy solutions to BVP (1.1) are obtained without the assumption for the Ambrosetti-Rabinowitz condition, while the A.R. condition is usually assumed in the literature. On the other hand, the assumed conditions in this paper are easy to verify, especially such as conditions $\left(\mathrm{H}_{2}\right)$ and $\left(\mathrm{H}_{4}\right)^{\prime}$. In general, some conditions corresponding to $\left(\mathrm{H}_{2}\right)$ and $\left(\mathrm{H}_{4}\right)^{\prime}$ are required to hold uniformly for some variable on some interval, while the formulas in $\left(\mathrm{H}_{2}\right)$ and $\left(\mathrm{H}_{4}\right)^{\prime}$ only need to hold for a.t. $t \in[0, T]$ due to applying Lemma 3.3 and Lemma 3.6 in this paper. So, it can be expected that Lemma 3.3 and Lemma 3.6 may be applied conveniently to some problems in the future to weaken some conditions. Finally, to show the effectiveness of the results obtained, two examples are given as follows.

Example 3.1 Consider the following boundary value problem:

$$
\left\{\begin{array}{l}
\frac{d}{d t}\left(\frac{1}{2} 0 D_{t}^{-\beta}\left(u^{\prime}(t)\right)+\frac{1}{2} t D_{T}^{-\beta}\left(u^{\prime}(t)\right)\right)+\nabla F(t, u(t))=0, \quad \text { a.e. } t \in[0, T] \\
u(0)=u(T)=0,
\end{array}\right.
$$

where ${ }_{0} D_{t}^{-\beta}$ and ${ }_{t} D_{T}^{-\beta}$ are the left and right Riemann-Liouville fractional integrals of order $0 \leq \beta<1$, respectively, $F(t, x)=\left(\sin \frac{\pi}{T} t\right)|x|^{\tau}+c|x|^{\theta}+e^{t} \ln \left(1+x^{2}\right), t \in[0, T], x \in \mathbb{R}$, and $\tau \in(1,2), \theta>2, c>0$.

It is easy to see that $F$ satisfies the following conditions:

(1) $F(t, x) \geq 0, F(t,-x)=F(t, x), t \in[0, T], x \in \mathbb{R}$.

(2) $F(t, x),\left|F_{x}(t, x)\right| \leq a(|x|) b(t), t \in[0, T], x \in \mathbb{R}$, where $a(x)=x^{\tau}+x^{\theta}+x^{2}+x^{\tau-1}+x^{\theta-1}+x$, $b(t)=\tau \sin \frac{\pi}{T} t+c \theta+2 e^{t}$.

(3) $\lim _{|x| \rightarrow \infty} \inf _{t \in[0, T]} \frac{F(t, x)}{|x|^{\theta}}=c>0, t \in[0, T]$.

(4) $F(t, x) \geq\left(\sin \frac{\pi}{T} t\right)|x|^{\tau}$ with $\tau \in(1,2)$ for $t \in[0, T], x \in \mathbb{R}$, and $0<\int_{0}^{T}\left(\sin \frac{\pi}{T} t\right)^{-\frac{1}{2}} d t<\infty$.

Also,

$$
F(t, x) \leq|x|^{\tau}+c|x|^{\theta}+e^{T} x^{2} \leq\left(1+c+e^{T}\right)|x|^{\tau}
$$

as $|x| \leq 1, t \in[0, T]$, noting that $\theta>2$, and $\tau \in(1,2)$.

(5) Take $\mu \in\left(\frac{1}{\theta}, \frac{1}{2}\right)$. Then $0<\mu \tau<1,1<\mu \theta$, and therefore,

$$
\begin{aligned}
& F(t, x)-\mu\left(F_{x}(t, x), x\right) \\
& \quad=\left(\sin \frac{\pi}{T} t\right)(1-\mu \tau)|x|^{\tau}+c(1-\mu \theta)|x|^{\theta}+e^{t}\left(\ln \left(1+x^{2}\right)-\frac{2 \mu}{1+x^{2}} x^{2}\right) \\
& \quad \leq\left(\sin \frac{\pi}{T} t\right)(1-\mu \tau)|x|^{\tau}+e^{T} \ln \left(1+x^{2}\right), \quad t \in[0, T], x \in \mathbb{R} .
\end{aligned}
$$


Owing to $\lim _{|x| \rightarrow 0} \frac{\ln \left(1+x^{2}\right)}{|x|^{\tau}}=0, \lim _{|x| \rightarrow \infty} \frac{\ln \left(1+x^{2}\right)}{|x|^{\tau}}=0$, there exists a $M>1$ such that $\ln \left(1+x^{2}\right) \leq$ $M|x|^{\tau}$, for all $x \in \mathbb{R}$. Hence,

$$
F(t, x)-\mu\left(F_{x}(t, x), x\right) \leq\left(1+M e^{T}\right)|x|^{\tau}, \quad t \in[0, T], x \in \mathbb{R} .
$$

So, all the assumptions of Theorem 3.1 are satisfied, and therefore, BVP (3.37) has infinitely many small energy solutions.

Example 3.2 Consider the following boundary value problem:

$$
\left\{\begin{array}{l}
\frac{d}{d t}\left(\frac{1}{2}{ }_{0} D_{t}^{-\beta}\left(u^{\prime}(t)\right)+\frac{1}{2} t D_{T}^{-\beta}\left(u^{\prime}(t)\right)\right)+\nabla F(t, u(t))=0, \quad \text { a.e. } t \in[0, T], \\
u(0)=u(T)=0
\end{array}\right.
$$

where ${ }_{0} D_{t}^{-\beta}$ and ${ }_{t} D_{T}^{-\beta}$ are the left and right Riemann-Liouville fractional integrals of order $0 \leq \beta<1$, respectively, $F(t, x)=e^{t}|x|^{p}+\eta \ln \left(1+x^{2} \sin ^{2} t\right), t \in[0, T], x \in \mathbb{R}, p>2$, and $\eta$ satisfying $0<\eta<\frac{\Gamma(\alpha+1)^{2}}{4 T^{2 \alpha}} \cos (\alpha \pi)$. It is easy to see that $F$ satisfies the following conditions:

(1) $F(t, x) \geq 0, F(t,-x)=F(t, x), t \in[0, T], x \in \mathbb{R}$.

(2) $F(t, x),\left|F_{x}(t, x)\right| \leq a(|x|) b(t), t \in[0, T], x \in \mathbb{R}$, where $a(x)=x^{p}+x^{p-1}+x+x^{2}, b(t)=$ $p e^{t}+2 \eta \sin ^{2} t$, noting that $\ln (1+x) \leq x$ for all $x \geq 0$.

(3) $F(t, x) \leq\left(e^{T}+\eta\right)\left(|x|^{p}+|x|^{2}\right)$ for all $t \in[0, T], x \in \mathbb{R}$.

(4) $\lim _{|x| \rightarrow 0} \frac{F(t, x)}{|x|^{2}} \leq \lim _{|x| \rightarrow 0} e^{T}|x|^{p-2}+\lim _{|x| \rightarrow 0} \eta \frac{\ln \left(1+x^{2} \sin ^{2} t\right)}{|x|^{2}}=\eta \sin ^{2} t \leq \eta, t \in[0, T]$.

(5) $\lim _{|x| \rightarrow \infty} \frac{F(t, x)}{|x|^{2}} \geq \lim _{|x| \rightarrow \infty}|x|^{p-2}=\infty$.

(6) Take $\mu \in(2, p)$. Then

$$
\begin{aligned}
\mu F(t, x)-\left(F_{x}(t, x), x\right) & =(\mu-p) e^{t}|x|^{p}+\mu \eta \ln \left(1+x^{2} \sin ^{2} t\right)-\frac{2 \eta x^{2} \sin ^{2} t}{1+x^{2} \sin ^{2} t} \\
& \leq \mu \eta \ln \left(1+x^{2} \sin ^{2} t\right) \\
& \leq \mu \eta\left(1+x^{2}\right)
\end{aligned}
$$

for all $t \in[0, T], x \in \mathbb{R}$. Hence, all the assumptions of Theorem 3.2 are satisfied and therefore BVP (3.38) has infinitely many high energy solutions.

Competing interests

The author declares that he has no competing interests.

\section{Author's contributions}

The author declares that he carried out all the work in this manuscript and read and approved the final manuscript.

\section{Acknowledgements}

The author sincerely thanks the anonymous referees for their valuable suggestions and comments which have greatly helped improve this article. This research is supported by the National Nature Science Foundation of China under grant 11601139.

Received: 19 February 2016 Accepted: 3 July 2016 Published online: 23 August 2016

\section{References}

1. Diethelm, K, Freed, AD: On the solution of nonlinear fractional order differential equations used in the modeling of viscoelasticity. In: Keil, F, Mackens, W, Voss, H, Werther, J (eds.) Scientific Computing in Chemical Engineering II Computational Fluid Dynamics, Reaction Engineering and Molecular Properties, pp. 217-224. Springer, Heidelberg (1999)

2. Lundstrom, BN, Higgs, MH, Spain, WJ, Fairhall, AL: Fractional differentiation by neocortical pyramidal neurons. Nat. Neurosci. 11, 1335-1342 (2008) 
3. Glockle, WG, Nonnenmacher, TF: A fractional calculus approach of self-similar protein dynamics. Biophys. J. 68, 46-53 (1995)

4. Hilfer, R: Applications of Fractional Calculus in Physics. World Scientific, Singapore (2000)

5. Mainardi, F: Fractional calculus: some basic problem in continuum and statistical mechanics. In: Carpinteri, A, Mainardi, F (eds.) Fractals and Fractional Calculus in Continuum Mechanics, pp. 290-349. Springer, Wien (1997)

6. Kilbas, AA, Srivastava, HM, Trujillo, JJ: Theory and Applications of Fractional Differential Equations. North-Holland Mathematics Studies, vol. 204. Elsevier, Amsterdam (2006)

7. Jiao, F, Zhou, Y: Existence of solutions for a class of fractional boundary value problems via critical point theory. Comput. Math. Appl. 62, 1181-1199 (2011)

8. Chen, J, Tang, X: Existence and multiplicity of solutions for some fractional boundary value problem via critical point theory. Abstr. Appl. Anal. 2012, Article ID 648635 (2012)

9. Chen, J, Tang, X: Infinitely many solutions for a class of fractional boundary value problem. Bull. Malays. Math. Sci. Soc 36, 1083-1097 (2013)

10. Kong, L: Existence of solutions to boundary value problems arising from the fractional advection dispersion equation Electron. J. Differ. Equ. 2013, 106 (2013)

11. Bin, G: Multiple solutions for a class of fractional boundary value problems. Abstr. Appl. Anal. 2012, Article ID 468980 (2012)

12. Zhang, $X, L i u, L, W u, Y:$ Variational structure and multiple solutions for a fractional advection-dispersion equation. Comput. Math. Appl. 68, 1794-1805 (2014)

13. Bai, C: Existence of three solutions for a nonlinear fractional boundary value problem via a critical points theorem. Abstr. Appl. Anal. 2012, Article ID 963105 (2012)

14. Li, Y, Sun, H, Zhang, Q: Existence of solutions to fractional boundary value problems with a parameter. Electron. J. Differ. Equ. 2013, 141 (2013)

15. Sun, $H$, Zhang, Q: Existence of solutions for a fractional boundary value problem via the mountain pass method and an iterative technique. Comput. Math. Appl. 64, 3436-3443 (2012)

16. Ferrara, M, Hadjian, A: Variational approach to fractional boundary value problems with two control parameters. Electron. J. Differ. Equ. 2015, 130 (2015)

17. Jiao, F, Zhou, Y: Existence results for fractional boundary value problems via critical point theory. Int. J. Bifurc. Chaos 22, $1250086(2012)$

18. Xie, W, Xiao, J, Luo, Z: Existence of solutions for fractional boundary value problem with nonlinear derivative dependence. Abstr. Appl. Anal. 2014, Article ID 812910 (2014)

19. Zhao, Y, Chen, H, Qin, B: Multiple solutions for a coupled system of nonlinear fractional differential equations via variational methods. Appl. Math. Comput. 257, 417-427 (2015)

20. Torres, C: Mountain pass solution for a fractional boundary value problem. J. Fract. Calc. Appl. 5, 1-10 (2014)

21. Benson, DA, Schumer, R, Meerschaert, MM, Wheatcraft, SW: Fractional dispersion, Lévy motion, and the MADE tracer test. Transp. Porous Media 42, 211-240 (2001)

22. Benson, DA, Wheatcraft, SW, Meerschaert, MM: Application of a fractional advection-dispersion equation. Water Resour. Res. 36, 1403-1412 (2000)

23. Benson, DA, Wheatcraft, SW, Meerschaert, MM: The fractional-order governing equation of Lévy motion. Water Resour. Res. 36, 1413-1423 (2000)

24. Zou, W: Variant fountain theorems and their applications. Manuscr. Math. 104, 343-358 (2001)

25. Zou, W, Schechter, M: Critical Point Theory and Its Applications. Springer, New York (2006)

26. Tang, C, Wu, X: Periodic solutions for second order systems with not uniformly coercive potential. J. Math. Anal. Appl. $259,386-397(2001)$

\section{Submit your manuscript to a SpringerOpen ${ }^{\circ}$ journal and benefit from:}

- Convenient online submission

Rigorous peer review

- Immediate publication on acceptance

Open access: articles freely available online

- High visibility within the field

- Retaining the copyright to your article 